\title{
Joint Emission Reduction Dynamic Decisions and Coordination in a Supply Chain Considering Altruistic Behavior and Reference Low-Carbon Effect
}

ziyuan zhang ( $\nabla$ zhangziyuan@shu.edu.cn )

Shanghai University

Liying Yu

Shanghai University

\section{Research Article}

Keywords: reference low-carbon effect,altruistic behavior,joint emission reduction,cost-sharing contract,differential game

Posted Date: May 24th, 2021

DOI: https://doi.org/10.21203/rs.3.rs-431496/v1

License: (c) (i) This work is licensed under a Creative Commons Attribution 4.0 International License.

Read Full License 


\title{
Joint Emission Reduction Dynamic Decisions and Coordination in a Supply Chain Considering Altruistic Behavior and Reference Low-Carbon Effect
}

\author{
Ziyuan Zhang ${ }^{1}$, Liying $\mathbf{Y u}^{1}$,* \\ 1 School of Management, Shanghai University, Shanghai 200444, China; zhangziyuan @ shu.edu.cn (Z.Z.) \\ ;yuliying@ @ shu.edu.cn (L.Y.) \\ * Correspondence: yuliying@ shu.edu.cn
}

\begin{abstract}
In the context of low-carbon economy, supply chain members' joint emission reduction issue has become a research hotspot, while there are few researches which synthetically studies the effect of consumers' reference low-carbon effect and supply chain members' altruistic behavior on their decisions. To study the impact of supply chain members' altruistic behavior and consumers' reference low-carbon effect on their joint emission reduction decisions and profits, we build optimization models under four decision scenarios, in which we solve the manufacturer's and the retailer's optimal emission reduction strategies and other equilibrium solutions by differential game theory. We obtain some findings. First, consumers' reference low-carbon effect will harm the profits of the manufacturer and the retailer, discourage the manufacturer's enthusiasm to reduce emissions and retailer's enthusiasm for low-carbon publicity. Second, the altruistic behavior of the manufacturer and the retailer can not only weaken the negative impact of the reference low-carbon effect, but also promote both parties to actively reduce emissions, help achieve Pareto improvement of their own profits and utilities, and obtain additional social welfare. Third, the cost-sharing contract can encourage the manufacturer to increase emission reduction investment without affecting the retailer's low-carbon publicity investment, and can achieve a Pareto improvement of both parties' profits and utilities. In addition, the cost-sharing ratio is only proportional to the marginal profits and altruistic intensity of both parties, and is not affected by the reference low-carbon effect. Meanwhile, the cost-sharing ratio will decrease as the manufacturer's marginal profit and altruistic intensity increase, and will increase as the retailer's marginal profit and altruistic intensity increase. In particular, when the retailer is completely altruistic, the cost-sharing contract can achieve perfect coordination of the supply chain.
\end{abstract}

Keywords: reference low-carbon effect; altruistic behavior; joint emission reduction; cost-sharing contract; differential game

\section{Introduction}

At present, facing the severe global climate change issue, reducing greenhouse gas emissions to reduce air pollution and greenhouse effect has become a global consensus, and a low-carbon economic development mode characterized by low pollution, low energy consumption and sustainable development has become the focus of attention of all countries. Subsequently, the European Union pledged in 2014 to reduce carbon emissions by $40 \%$ from 1990 levels by 2030. China promised to reduce carbon emissions per unit of GDP by $40 \%$ to $50 \%$ by 2020 (compared to 2005). Meanwhile, affected by the deterioration of the ecological environment, the public's awareness of environmental protection has been further improved, more and more consumers are willing to buy low-carbon products (Zhang et al.2015). According to a consumer survey, $80 \%$ of American consumers would like to buy low-carbon products even though they have to pay an additional fee (Zhang et al.2020). Similarly, more and more Chinese consumers are willing to purchase low-carbon products on the Ali platform, and the compound growth rate of annual sales of low-carbon products was greater than $80 \%$ between 2011 and 2015 (Xia et al.2020). In the context of low-carbon economy, to obtain sustainable competitive advantages, actively carry out emission reduction activities will be an important way for companies around the world.

Facing the pressure of emission reduction and market competition, more and more manufactures in the supply chain have also started to cooperate with retailers to carry out emission reduction 
activities. On the one hand, manufacturers produce low-carbon products with higher low-carbon level by increasing emission reduction investment and strengthening the low-carbon technology research and development. For instance, in order to reduce carbon emissions, Haier, a home appliance manufacturer that has been committed to practicing the low-carbon concept. Since the launch of the photovoltaic power generation project, it has successively launched energy-saving and low-carbon central air conditioners with a haze removal rate of $99.8 \%$ and above. On the other hand, retailers implement low-carbon promotion to draw consumers in the market to buy low-carbon products. For instance, JD and Taobao in China promote low-carbon products by using poster display and merchandise exhibition. Wal-Mart requires its suppliers to increase their emissions reduction investment and join the carbon emission disclosure program as required. At the same time, it will also use its strong network to provide special product promotion for the suppliers in the plan (Zhou et al.2016). Many examples in reality and a large number of previous studies have shown that manufacturers and retailers conducting joint emission reduction activities has been an effective way when one party faces greater pressure to reduce emissions. Thus, discussing how supply chain members can effectively carry out joint emission reductions will be of great significance.

In the process of practicing low-carbon economy, consumers' low-carbon consumption awareness is a factor that cannot be ignored for companies. Companies must fully study consumers' behavior characteristics and carefully consider them when making operational decisions to obtain stronger market competitiveness and greater market share. After a lot of researches, scholars find that when consumers are going to buy products, both the current attributes and the expected attributes formed by their previous purchase experiences will affect their purchasing behavior. This kind of purchasing psychology is defined as reference effect by scholars (Dye et al.2018; Liu et al.2016). Accordingly, when consumers choose to buy low-carbon products, they will not only be affected by the present low-carbon level of the products, but also be affected by the reference point of low-carbon level formed from their past purchase experience. Based on the definition of the reference effect, we define it as reference low-carbon effect. Due to the significant impact of the reference low-carbon effect on consumers' low-carbon buying behavior, it is very necessary to introduce it into the issue of joint emission reduction and study its impact on supply chain members' emission reduction decisions.

In addition, many behavioral economics experiments and studies have shown that people not only pay attention to their own interests, but also the interests of partners in the transaction process, which is manifested as altruistic attributes (Loch and Wu.2008). Ignoring the altruistic behavior of individuals, the research results often deviate from reality when researches are based on individual rationality (Bendoly et al.2006). At present, with the continuous development of economic globalization, altruistic behavior among supply chain members is becoming more and more obvious. For example, IBM establishes the "IBM China Channel University" to share the latest technology and management experience with channel partners; General Motors in the United States contributes funds to help its suppliers achieve technological upgrades, and Toyota provides a large amount of technical and management support for its parts suppliers to help them improve product productivity; Lenovo helps its channel provider Teng Chuang Technology to raise funds to achieve a win-win situation. There is no doubt that with the development of globalization, supply chain members will pay more and more attention to mutual benefit with their partners, and their altruistic preferences will become more and more obvious, which will have a significant impact on their decision-making. Therefore, in the context of a low-carbon economy, it is of great practical significance to explore the impact of supply chain members' altruistic behavior on their cooperative emission reduction decisions.

Under the above background and based on Stackelberg differential game method, this paper explores the issue of joint emission reduction of supply chain with the consideration of supply chain members' altruistic behavior and consumers' reference low-carbon effect from a long-term perspective. The following problems will be solved in this paper.

Under the impact of supply chain members' altruistic behavior and consumers' reference low-carbon effect and different scenarios, what are the supply chain members' equilibrium strategies.

What are the optimal evolution paths for product low-carbon level, reference low-carbon level and low-carbon goodwill over time under different scenarios? 
what impact will the cost-sharing contract have on the supply chain members' equilibrium strategies?

What conditions should be met to achieve supply chain coordination with or without cost-sharing contract if supply chain members have altruistic behavior.

By answering the above questions, this paper mainly has the following contributions.

First, this paper simultaneously integrates altruistic behavior and consumers' reference low-carbon effect these two factors to study supply chain members' joint emission reduction decisions and supply chain coordination. Second, this paper establishes supply chain emission reduction dynamic optimization models under four different decision scenarios, and obtains the joint emission reduction strategies of supply chain members from a long-term perspective. In short, through mathematical derivation and numerical analysis, this paper has deeply discussed the impact of these two factors on the supply chain members' joint emission reduction decisions, and can provide theoretical support for supply chain members' long-term cooperative emission reduction under different decision scenarios.

This paper is organized as follows. Section 2 presents the literature review. Section 3 provides problem description. The equilibrium solutions under four decision scenarios are provided in Section 4. Section 5 is the analysis and comparison section. Section 6 gives some numerical examples to verify the findings. Section 7 summarizes conclusions and limitations of this paper.

\section{Literature Review}

Based on the content of our research, there are two main streams of literature related to the issue of supply chain operation that we will need to review. One is the issue of joint emission reduction in the supply chain considering altruistic behavior or not, another one is the issue of operational decision in the supply chain with consideration of consumers' reference effect.

Currently, joint emission reduction issue in the supply chain has been deeply studied in the context of low-carbon economy (Benjaafar et al.2013). Considering consumers' low-carbon preference and a retailer-led supply chain, the emission reduction and coordination issue was studied by Wang et al. (2016). Liu et al. (2021) studied carbon emission reductions and coordination in the supply chain considering consumers' strong low-carbon preference. With the consideration of consumers' low-carbon preference, $\mathrm{Pu}$ et al. (2018) studied the impact of government policy and supply chains' competition. Considering supply chain members' fairness concern behavior, Li et al.(2018) studied pricing decisions and emission reduction strategies in the supply chain, then pointed out a contract to achieve the coordination of the supply chain. $\mathrm{Yu}$ and Hou (2021) considered stochasticity and consumers' low-carbon preference and studied supply chain members' emission reduction decisions. In a low-carbon e-supply chain, Han et al. (2018) discussed the manufacturer's emission reduction decisions. Zhang et al. (2018) considered the retailer's fairness concern and government subsidies, and studied low-carbon supply chain members' emission reduction decisions. Wang et al. (2020) explored emission reduction decisions and production decisions with the consideration of cap-and-trade policy, then used the two-way cost-sharing contract to realize the coordination under cap-and-trade regulation policy. Considering a dual-channel low-carbon supply chain and consumers' low-carbon preference, Zhang et al. (2020) studied supply chain members' emission reduction decisions. Zhang et al. (2021) considered a capital-constrained supply chain, and studied the impact of quity financing and consumers' low-carbon preference on supply chain members' emission reduction decisions. Considering financial constraints, Cao et al. (2020) studied low-carbon supply chain members' carbon reduction decisions and product order quantity decisions.

Although the above-mentioned researches have studied the emission reduction issues of the supply chain from different perspectives such as consumers' low-carbon preference, government regulations, supply chain competition and coordination, they are all based on the premise that the decision makers are completely rational, and have not considered supply chain members' altruistic behavior.

As the researches relevant to altruistic behavior continue to deepen, discussing the impact of altruistic behavior in the issue of supply chain joint emission reduction has gradually attracted the attention of scholars. Huang et al. (2019) established four models: two cooperative manufacturers and one retailer are completely self-interested, one retailer has altruistic preference, two cooperative 
manufacturers have altruistic preferences, then studied the impact of supply chain members' altruistic behavior on their emission reduction and pricing decisions. Considering the carbon tax policy and the altruistic preference of supply chain members, Cheng et al. (2018) established the supply chain pricing and emission reduction decision models under the condition of complete self-interest, retailer's altruistic preference and manufacturer's altruistic preference, and analyzed the influence of supply chain altruistic preference attributes on the decision-making of supply chain members. Xia et al. (2018) introduced reciprocal preferences and consumers' low-carbon awareness into the dyadic supply chain, and investigated how reciprocity and CLA affect the emission reduction decisions and performances of the supply chain members.

Although the above studies discuss the impact of altruistic behavior of supply chain members on their emission reduction decisions, the emission reduction optimization models they establish are all static optimization models. The strategies got by the static decision-making models can just report the supply chain members' short-term optimal decisions, but the dynamic decision-making models can better report the long-term effects of supply chain members' emission reductions. At present, there are few literatures that consider the altruistic behavior of supply chain members and use dynamic optimization models to discuss the issue of joint emission reduction in the supply chain. As far as we know, only Xia et al. (2020) studied joint emission reduction issues by using differential game theory considering social preferences of supply chain members. However, Xia et al. (2020) only considered supply chain members' social preferences in the emission reduction model, they do not consider consumers' reference low-carbon effect and product low-carbon goodwill, simultaneously. Ignoring consumers' reference low-carbon effect and product low-carbon goodwill will inevitably cause models and research conclusions to deviate from reality, so the research content needs to be supplemented.

Consumer behavior studies have shown that the difference between the actual product attribute level experienced by consumers after shopping and the product reference attribute level expected by consumers before shopping will have an important impact on product goodwill and market demand, that is, consumers' reference effect. Recently, there have been some studies that introduce consumers' reference effect into the issue of supply chain operation decisions. considering reference price effect, Popescu et al. (2007) studied the issue of dynamic pricing decisions. Considering consumers' reference price effect and the advertising effect, Zhang et al. (2013) designed a bilateral cost-sharing contract to realize the supply chain coordination. Considering reference price effect, Lin (2016) studied the issue of price promotion in supply chain. Based on the situation of supply chain competition, Zhang et al. (2014) considered the reference price effect and studied strategic pricing decisions of supply chain members. Considering reference quality effect, Liu et al. (2016) discussed supply chain members' myopic and far-sighted behaviors. Zhang et al. (2018) studied supply chain members' static and dynamic pricing decisions in a closed-loop supply chain with the consideration of reference quality effect. In the context of the $\mathrm{O} 2 \mathrm{O}$ environment, $\mathrm{He}$ et al. (2018) explored supply chain members' equilibrium strategies considering reference quality effect.

The above literature shows that reference price effect and reference quality effect have been extensively and in-depth studied, but there are still few studies on consumers' reference low-carbon effect. With the growing prosperity of the low-carbon economy, the awareness of low-carbon consumption has become increasingly popular, there is no doubt that consumers' reference low-carbon effect will have a remarkable influence on their low-carbon consumption behavior. At the same time, as supply chain members carrying out joint emission reduction activities is becoming increasingly common, studying the joint emission reduction issue considering their altruistic behavior will be very necessary. In Table 1, we have summarized some literatures that have strong relevance to this paper to further reveal the innovation and contribution of this paper.

Table 1 The main contributions of this paper

\begin{tabular}{ccccc}
\hline Author & $\begin{array}{c}\text { Reference } \\
\text { Low-Carb } \\
\text { on Effect }\end{array}$ & $\begin{array}{c}\text { Altruistic } \\
\text { Behavior }\end{array}$ & $\begin{array}{c}\text { Low Carbon } \\
\text { Goodwill }\end{array}$ & $\begin{array}{c}\text { Supply Chain } \\
\text { Coordination }\end{array}$ \\
\hline Lou et al. (2018) & $\checkmark$ & & & $\checkmark$ \\
Xia et al. (2020) & & $\checkmark$ & & $\checkmark$
\end{tabular}


et al. (2017)

Fan et al. (2019)

Wang et al. (2020)

Zhang and $\mathrm{Yu}$ (2021)

This paper

Therefore, taking into account the shortcomings of the previous literature and considering the impact of supply chain members' altruistic behavior and consumers' reference low-carbon effect, this paper will study how these two factors impact the supply chain members' optimal emission reduction strategies, profits and utilities under different decision scenarios through the differential game theory. Furthermore, we will also explore the conditions for the cost-sharing contract to achieve supply chain coordination when supply chain members have altruistic behavior.

\section{Problem Description}

To simplify the model and highlight the research focus, we consider a supply chain system including a manufacturer and a retailer. The manufacturer produces a sort of low-carbon product by emission reduction investment, and the low-carbon level of the product will be higher when the manufacturer's emission reduction level is higher. Meanwhile, the retailer will also implement low-carbon promotion will also be implemented by the retailer to draw consumers to purchase this sort of low-carbon product. In addition, since manufacturers often need to pay greater emission reduction costs to reduce emissions, retailers often share their emission reduction costs to encourage manufacturers to reduce emissions. Some related parameters and decision variables involved in this paper are explained in Table 2.

To better serve follow-up research, we propose some assumptions as follows:

Assumption 1 It is assumed that the manufacturer is the leader and the retailer is the follower in the supply chain system. Both manufacturers and retailers grasp symmetrical and clear market information. When they do not have altruistic behavior, they play Stackelberg game to maximize their own profits. But when they have altruistic behavior, both of them will play Stackelberg game to maximize their own altruistic utilities.

Assumption 2 Manufacturers can increase the low-carbon level of their products by investing in emission reductions, and due to various factors such as equipment aging and backward technology, the low-carbon level of the product has a natural decline over time. Learn from previous literature(Bertinelli et al.2014), we use the differential equation shown in equation (1) to describe the dynamic change process of the product's low carbon level over time.

$$
\begin{aligned}
& \dot{T}(t)=\gamma E(t)-\delta T(t) \\
& T(0)=T_{0}
\end{aligned}
$$

Assumption 3 According to Hellofs et al.(1999), before purchasing low-carbon products, consumers will make a basic judgment on the low-carbon level of the product based on the product's low-carbon goodwill, and believe that the product's reference low-carbon level is positively correlated with its low-carbon goodwill. Therefore, we follow Hellofs's point of view and assume that the product reference low-carbon level and low-carbon goodwill have the following relationship:

$$
\begin{aligned}
& R(t)=\varepsilon G(t) \\
& R(0)=\varepsilon G_{0}=R_{0}
\end{aligned}
$$

Assumption 4 Refer to He et al.(2017), assuming that the product's low-carbon goodwill is affected by both the consumer's reference low-carbon effect and the retailer's low-carbon publicity investment. When the actual low-carbon level of the product purchased by the consumer is higher than the reference low-carbon level, the low-carbon goodwill will be improved. When the actual low-carbon level of the product purchased by consumers is lower than expected, it will have a negative impact on the low-carbon goodwill of the product. Following the point of view of He et al. (2017) and based on the classic goodwill model of Nerlove-Arrow, the product low-carbon goodwill function is shown as equation (3). 


$$
\begin{aligned}
& \dot{G}(t)=\phi[T(t)-R(t)]+\lambda A(t)-\sigma G(t) \\
& G(0)=G_{0}
\end{aligned}
$$

Substituting equation (2) in Assumption 3 into equation (3) in Assumption 4, and let $\vartheta=\phi \varepsilon+\sigma$, we can further obtain the dynamic expression of low-carbon goodwill as shown in equation (4).

$$
\begin{aligned}
& \dot{G}(t)=\phi T(t)+\lambda A(t)-\vartheta G(t) \\
& G(0)=G_{0}
\end{aligned}
$$

Assumption 5 According to the literature (Kopalle and Winer 1996), we assume that the low-carbon product's demand function is a deterministic and linear function. Products with higher low-carbon level, low-carbon promotion level and low-carbon goodwill level are most popular among consumers. At the same time, consumers will choose to purchase such product when its reference low-carbon level is lower than the actual low-carbon level at present, otherwise, they will be reluctant to buy the product because of the impact of reference low-carbon effect. What needs special explanation is that with the current product prices becoming more and more transparent, consumers are no longer as sensitive to product prices as they used to be. Instead, they pay more attention to non-price factors. Therefore, this paper does not consider the impact of product prices. The low-carbon product's demand function is shown as equation (5).

$$
Q=v+\alpha[T(t)-R(t)]+\mu G(t)+k A(t)
$$

Assumption 6 Refer to the literature (Ghosh and Shah 2015), we assume that the emission reduction cost of the manufacturer is $C_{m}=\frac{1}{2} \eta_{m} E^{2}(t)$, the low-carbon promotion cost of the retailer is $C_{r}=\frac{1}{2} \eta_{r} A^{2}(t)$

Assumption 7 Without considering the impact of price, the manufacturer's marginal revenue is assumed to be $\pi_{1}$, the retailer's marginal revenue is assumed to be $\pi_{2} . \pi_{1}$ and $\pi_{2}$ are both assumed to be constants. It is also assumed that both parties have the equal discount rate $\rho(\rho>0)$ at any time during a business scope of unlimited time. Besides, the transportation and inventory costs of the product will not be considered.

Table 2. Related parameters and decision variables involved in this paper

\begin{tabular}{cr}
\hline $\begin{array}{c}\text { Decision } \\
\text { Variables }\end{array}$ & Definition \\
\hline$E(t)$ & Manufacturer's emission reduction investment at time $t$ \\
$A(t)$ & Retailer's low-carbon promotion investment at time $t$ \\
\hline Parameters & Definition \\
\hline$\alpha$ & Influence coefficient of reference low carbon effect on the demand, $\alpha>0$ \\
$\gamma$ & Potential market demand for low-carbon products, $v>0$ \\
$\delta$ & Manufacturer's emission reduction efficiency coefficient, $\gamma>0$ \\
$k$ & Natural decay rate of product's low-carbon level, $\delta>0$ \\
$\rho$ & Correlation coefficient between reference low carbon level and low carbon goodwill, \\
$\mu$ & $\varepsilon>0$ \\
$\phi$ & Influence coefficient of low-carbon goodwill on the demand, $\mu>0$ \\
$\lambda$ & Influence coefficient of retailers' low-carbon publicity on low-carbon goodwill, $\lambda>0$ \\
$\sigma$ & Natural decay rate of product's low-carbon goodwill, $\sigma>0$ \\
$\pi_{1}$ & Manufacturer's marginal revenue, $\pi_{1}>0$
\end{tabular}




$\begin{array}{cc}U_{m} & \text { Manufacturer's utility } \\ \pi_{2} & \text { Retailer's marginal revenue, } \pi_{2}>0 \\ U_{r} & \text { Retailer's utility } \\ G(t) & \text { Low-carbon goodwill level at time } t \\ R(t) & \text { Reference low carbon level at time } t \\ T(t) & \text { Low-carbon level at time } t \\ G_{0} & \text { Initial low-Carbon Goodwill Level, } G_{0} \geq 0 \\ R_{0} & \text { Initial reference low carbon level, } R_{0} \geq 0 \\ T_{0} & \text { Initial low-carbon level, } T_{0} \geq 0 \\ \tau_{m} & \text { Manufacturer's altruistic preference value } \\ \tau_{r} & \text { Retailer's altruistic preference value } \\ \xi & \text { Cost-sharing rate }\end{array}$

$\eta_{m} 、 \eta_{r} \quad$ Emission reduction cost coefficient and low-carbon promotion cost coefficient.

\section{Model Solution}

According to the research assumptions in the former section, in order to conduct a comparative analysis of altruistic behavior, this section will first analyze the strategies and performances of the members under centralized scenario and decentralized scenario without altruistic behavior. Then this section will analyze decentralized scenario with altruistic behavior and decentralized scenario with altruistic behavior and a cost-sharing contract these two decision scenarios. In order to distinguish among different scenarios, superscripts $c, d, a, y$ respectively represent centralized decision scenario, decentralized decision scenario without altruistic behavior, decentralized decision scenario with altruistic behavior, decentralized decision scenario with altruistic behavior and a cost-sharing contract these four decision scenarios. In addition, subscripts $m, r, s c$ respectively represent the manufacturer, retailer and the supply chain system these three decision makers. For the convenience of writing, the time will not be listed below. Here, the Hamilton-Jacobi-Bellman equation which also expressed as the HJB equation is used to solve the equilibrium solutions of the different models.

\subsection{Centralized Scenario}

To achieve the optimal profit of the supply chain system, both the manufacturer and the retailer will jointly determine their emission reduction investment decisions. Although the centralized decision scenario is the most ideal supply chain decision scenario, it is difficult to achieve in actual enterprise operations. Here we use it as the upper limit of reference for analyzing altruistic behavior. At this time, the supply chain system's objective function is as follows:

$$
J_{s c}^{c}(T, G, t)=\max _{E, A} \int_{0}^{\infty} e^{-\rho t}\left[\left(\pi_{1}+\pi_{2}\right)[v+\alpha T+k A+(\mu-\alpha \varepsilon) G]-\frac{\eta_{m} E^{2}}{2}-\frac{\eta_{r} A^{2}}{2}\right] d t
$$

$$
\text { s.t. } \dot{T}(t)=\gamma E(t)-\delta T(t), T(0)=T_{0} \geq 0
$$

$$
\dot{G}(t)=\phi T(t)+\lambda A(t)-\vartheta G(t), G(0)=G_{0} \geq 0
$$

Theorem 1 (1) The manufacturer's and retailer's optimal emission reduction decisions are as follows:

$$
\left\{\begin{array}{l}
E^{c^{*}}=\frac{\gamma\left(\pi_{1}+\pi_{2}\right)[\phi(\mu-\alpha \varepsilon)+\alpha(\rho+\vartheta)]}{\eta_{m}(\rho+\vartheta)(\rho+\delta)} \\
A^{c^{*}}=\frac{\left(\pi_{1}+\pi_{2}\right)[\lambda(\mu-\alpha \varepsilon)+k(\rho+\vartheta)]}{\eta_{r}(\rho+\vartheta)}
\end{array}\right.
$$




$$
T^{c}(t)=\left(T_{0}-T_{\infty}^{c}\right) e^{-\delta t}+T_{\infty}^{c}
$$

Where $T_{\infty}^{c}=\frac{\gamma^{2}\left(\pi_{1}+\pi_{2}\right)[\phi(\mu-\alpha \varepsilon)+\alpha(\rho+\vartheta)]}{\delta \eta_{m}(\rho+\vartheta)(\rho+\delta)}$.

(3) The optimal evolution paths of low-carbon goodwill and reference low carbon level are as follows: :

$$
G^{c}(t)=\left(G_{0}+B^{c}-G_{\infty}^{c}\right) e^{-\vartheta t}-B^{c} e^{-\delta t}+G_{\infty}^{c}
$$

Where $B^{c}=\frac{\phi T_{0}}{\delta-\vartheta}-\frac{\phi \gamma^{2}\left(\pi_{1}+\pi_{2}\right)[\phi(\mu-\alpha \varepsilon)+\alpha(\rho+\vartheta)]}{\delta \eta_{m}(\rho+\vartheta)(\rho+\delta)(\delta-\vartheta)}$,

$$
G_{\infty}^{c}=\frac{\phi \gamma^{2}\left(\pi_{1}+\pi_{2}\right)[\phi(\mu-\alpha \varepsilon)+\alpha(\rho+\vartheta)]}{\delta \vartheta \eta_{m}(\rho+\vartheta)(\rho+\delta)}+\frac{\lambda\left(\pi_{1}+\pi_{2}\right)[\lambda(\mu-\alpha \varepsilon)+k(\rho+\vartheta)]}{\vartheta \eta_{r}(\rho+\vartheta)} .
$$

(4) The optimal total profit of the supply chain system is as follows:

$$
\pi_{s c}^{c}(t)=\pi_{\infty}^{c}+a_{1}^{*}\left(T_{0}-T_{\infty}^{c}\right) e^{-\delta t}+b_{1}^{*}\left[\left(G_{0}+B^{c}-G_{\infty}^{c}\right) e^{-\vartheta t}-B^{c} e^{-\delta t}\right]
$$

Where $\pi_{\infty}^{c}=a_{1}^{*} T_{\infty}^{c}+b_{1}^{*} G_{\infty}^{c}+c_{1}^{*}, a_{1}^{*}=\frac{\left(\pi_{1}+\pi_{2}\right)[\phi(\mu-\alpha \varepsilon)+\alpha(\rho+\vartheta)]}{(\rho+\vartheta)(\rho+\delta)} \square b_{1}^{*}=\frac{(\mu-\alpha \varepsilon)\left(\pi_{1}+\pi_{2}\right)}{\rho+\vartheta}$, $c_{1}^{*}=\frac{1}{\rho}\left\{\left(\pi_{1}+\pi_{2}\right) v+\frac{\gamma^{2}\left(\pi_{1}+\pi_{2}\right)^{2}[\phi(\mu-\alpha \varepsilon)+\alpha(\rho+\vartheta)]^{2}}{2 \eta_{m}(\rho+\vartheta)^{2}(\rho+\delta)^{2}}+\frac{\left(\pi_{1}+\pi_{2}\right)^{2}[\lambda(\mu-\alpha \varepsilon)+k(\rho+\vartheta)]^{2}}{2 \eta_{r}(\rho+\vartheta)^{2}}\right\}$

\section{The proof of Theorem 1 is similar to the proof of Theorem 3 and is omitted.}

Theorem 1 shows that the manufacturer's equilibrium emission reduction investment, the retailer's equilibrium low-carbon publicity investment, the steady-state low-carbon level, the steady-state low-carbon goodwill and the overall profit of the supply chain are all positively correlated with the marginal profits of the manufacturer and the retailer. This means that in order to further highlight the advantages of centralized decision-making and improve the overall profit level of the supply chain, manufacturers and retailers should take a variety of measures to increase their own marginal profits. For example, manufacturers can improve production efficiency, reduce production costs and transportation costs through technological upgrades, and retailers can improve marketing efficiency through accurate advertising. In addition, both parties should strengthen communication and cooperation, improve the overall operating efficiency of the supply chain and reduce the overall operating costs of the supply chain, thereby further improving the overall performance of the supply chain.

\subsection{Decentralized Scenario}

Under decentralized decision scenario, both the manufacturer and the retailer aim at maximizing their own profits to make decisions. This decision-making model is often used in the actual operation of enterprises, but the inherent double marginal effect of the decentralized decision-making model often leads to the loss of the supply chain system's profit. Therefore, here, we will use it as the lower limit of the reference for analyzing altruistic behavior.

At this time, the manufacturer's objective function is: 


$$
J_{m}^{d}(T, G, t)=\max _{E} \int_{0}^{\infty} e^{-\rho t}\left[\pi_{1}[v+\alpha T+k A+(\mu-\alpha \varepsilon) G]-\frac{\eta_{m} E^{2}}{2}\right] d t
$$

$$
\text { s.t. } \dot{T}(t)=\gamma E(t)-\delta T(t), T(0)=T_{0} \geq 0
$$

$$
\dot{G}(t)=\phi T(t)+\lambda A(t)-\vartheta G(t), G(0)=G_{0} \geq 0
$$

The retailer's objective function is:

$$
\begin{aligned}
& J_{r}^{d}(T, G, t)=\max _{A} \int_{0}^{\infty} e^{-\rho t}\left[\pi_{2}[\nu+\alpha T+k A+(\mu-\alpha \varepsilon) G]-\frac{\eta_{r} A^{2}}{2}\right] d t \\
& \text { s.t. } \dot{T}(t)=\gamma E(t)-\delta T(t), T(0)=T_{0} \geq 0 \\
& \dot{G}(t)=\phi T(t)+\lambda A(t)-\vartheta G(t), G(0)=G_{0} \geq 0
\end{aligned}
$$

Theorem 2 (1) The manufacturer's and retailer's optimal emission reduction decisions are as follows:

$$
\left\{\begin{array}{l}
E^{d^{*}}=\frac{\gamma \pi_{1}[\phi(\mu-\alpha \varepsilon)+\alpha(\rho+\vartheta)]}{\eta_{m}(\rho+\vartheta)(\rho+\delta)} \\
A^{d^{*}}=\frac{\pi_{2}[k(\rho+\vartheta)+\lambda(\mu-\alpha \varepsilon)]}{\eta_{r}(\rho+\vartheta)}
\end{array}\right.
$$

(2) The optimal evolution path of low-carbon level is as follows:

$$
T^{d}(t)=\left(T_{0}-T_{\infty}^{d}\right) e^{-\delta t}+T_{\infty}^{d}
$$

Where $T_{\infty}^{d}=\frac{\gamma^{2} \pi_{1}[\phi(\mu-\alpha \varepsilon)+\alpha(\rho+\vartheta)]}{\delta \eta_{m}(\rho+\vartheta)(\rho+\delta)}$

(3) The optimal evolution paths of low-carbon goodwill and reference low carbon level are as follows: :

$$
G^{d}(t)=\left(G_{0}+B^{d}-G_{\infty}^{d}\right) e^{-\vartheta t}-B^{d} e^{-\delta t}+G_{\infty}^{d}
$$

$$
R^{d}(t)=\varepsilon G^{d}(t)
$$

Where $B^{d}=\frac{\phi T_{0}}{\delta-\vartheta}-\frac{\phi \gamma^{2} \pi_{1}[\phi(\mu-\alpha \varepsilon)+\alpha(\rho+\vartheta)]}{\delta \eta_{m}(\rho+\vartheta)(\rho+\delta)(\delta-\vartheta)}$,

$G_{\infty}^{d}=\frac{\phi \gamma^{2} \pi_{1}[\phi(\mu-\alpha \varepsilon)+\alpha(\rho+\vartheta)]}{\delta \vartheta \eta_{m}(\rho+\vartheta)(\rho+\delta)}+\frac{\lambda \pi_{2}[\lambda(\mu-\alpha \varepsilon)+k(\rho+\vartheta)]}{\vartheta \eta_{r}(\rho+\vartheta)}$.

(4) The optimal profits of the manufacturer and retailer are as follows:

$$
\begin{aligned}
& \pi_{m}^{d}(t)=\pi_{m \infty}^{d}+a_{3}^{*}\left(T_{0}-T_{\infty}^{d}\right) e^{-\delta t}+b_{3}^{*}\left[\left(G_{0}+B^{d}-G_{\infty}^{d}\right) e^{-\vartheta t}-B^{d} e^{-\delta t}\right] \\
& \pi_{r}^{d}(t)=\pi_{r \infty}^{d}+a_{2}^{*}\left(T_{0}-T_{\infty}^{d}\right) e^{-\delta t}+b_{2}^{*}\left[\left(G_{0}+B^{d}-G_{\infty}^{d}\right) e^{-\vartheta t}-B^{d} e^{-\delta t}\right]
\end{aligned}
$$

Where $\pi_{m \infty}^{d}=a_{3}^{*} T_{\infty}^{d}+b_{3}^{*} G_{\infty}^{d}+c_{3}^{*}, \pi_{r \infty}^{d}=a_{2}^{*} T_{\infty}^{d}+b_{2}^{*} G_{\infty}^{d}+c_{2}^{*}, a_{2}^{*}=\frac{\pi_{2}[\phi(\mu-\alpha \varepsilon)+\alpha(\rho+\vartheta)]}{(\rho+\vartheta)(\rho+\delta)}$, 
$b_{2}^{*}=\frac{\pi_{2}(\mu-\alpha \varepsilon)}{\rho+\vartheta} \quad, \quad a_{3}^{*}=\frac{\pi_{1}[\phi(\mu-\alpha \varepsilon)+\alpha(\rho+\vartheta)]}{(\rho+\vartheta)(\rho+\delta)} \quad, \quad b_{3}^{*}=\frac{\pi_{1}(\mu-\alpha \varepsilon)}{\rho+\vartheta}$,

$c_{2}^{*}=\frac{1}{\rho}\left\{\pi_{2} v+\frac{\gamma^{2} \pi_{1} \pi_{2}[\phi(\mu-\alpha \varepsilon)+\alpha(\rho+\vartheta)]^{2}}{\eta_{m}(\rho+\vartheta)^{2}(\rho+\delta)^{2}}+\frac{\pi_{2}^{2}[(\rho+\vartheta) k+\lambda(\mu-\alpha \varepsilon)]^{2}}{2 \eta_{r}(\rho+\vartheta)^{2}}\right\}$,

$c_{3}^{*}=\frac{1}{\rho}\left\{\pi_{1} \nu+\frac{\gamma^{2} \pi_{1}^{2}[\phi(\mu-\alpha \varepsilon)+\alpha(\rho+\vartheta)]^{2}}{2 \eta_{m}(\rho+\vartheta)^{2}(\rho+\delta)^{2}}+\frac{\pi_{1} \pi_{2}[(\rho+\vartheta) k+\lambda(\mu-\alpha \varepsilon)]^{2}}{\eta_{r}(\rho+\vartheta)^{2}}\right\}$.

The proof of Theorem 2 is similar to the proof of Theorem 3 and is omitted.

Theorem 2 shows that the manufacturer's emission reduction investment has a positive correlation with its marginal revenue and a negative relationship with its emission reduction cost coefficient. The retailer's low-carbon publicity investment also has a positive correlation with its marginal revenue and a negative correlation with its low-carbon publicity cost coefficient. This shows that under decentralized decision scenario, manufacturers and retailers often make decisions based on their own interests, while ignoring the overall benefits of the supply chain, which will result in a double marginal effect and damage the overall profit of the supply chain. In the face of this situation, first of all, both parties should abandon individualism and think more from the perspective of the overall supply chain. On the other hand, they should actively improve their own marginal revenue through various measures such as technological upgrades and accurate advertising.

\subsection{Decentralized Scenario with Altruistic Behavior}

When manufacturers and retailers have altruistic behavior, they will not only pay attention to their own profits, but also pay attention to the profits of the other party. That is, both parties will make decisions with the goal of maximizing their own utility. Referring to the construction of the altruistic utility function of Bassi et al.(2014) in the static category, this section extends the altruistic utility function to the dynamic category, so the altruistic utility functions of the manufacturer and retailer are as follows:

$$
\begin{gathered}
U_{m}(t)=\pi_{m}(t)+\tau_{m} \pi_{r}(t) \\
U_{r}(t)=\pi_{r}(t)+\tau_{r} \pi_{m}(t)
\end{gathered}
$$

At this time, the manufacturer's objective function is:

$$
\begin{aligned}
& J_{m}^{a}(T, G, t)=\max _{E} \int_{0}^{\infty} e^{-\rho t}\left[\pi_{1}\left[\begin{array}{l}
v+\alpha T+k A \\
+(\mu-\alpha \varepsilon) G
\end{array}\right]+\tau_{m}\left\{\pi_{2}\left[\begin{array}{l}
v+\alpha T+k A \\
+(\mu-\alpha \varepsilon) G
\end{array}\right]-\frac{\eta_{r} A^{2}}{2}\right\}-\frac{\eta_{m} E^{2}}{2}\right] d t \\
& \text { s.t. } \dot{T}(t)=\gamma E(t)-\delta T(t), T(0)=T_{0} \geq 0 \\
& \dot{G}(t)=\phi T(t)+\lambda A(t)-\vartheta G(t), G(0)=G_{0} \geq 0
\end{aligned}
$$

The retailer's objective function is:

$$
\begin{aligned}
& J_{r}^{a}(T, G, t)=\max _{A} \int_{0}^{\infty} e^{-\rho t}\left[\pi_{2}\left[\begin{array}{l}
v+\alpha T+k A \\
+(\mu-\alpha \varepsilon) G
\end{array}\right]+\tau_{r}\left\{\pi_{1}\left[\begin{array}{l}
v+\alpha T+k A \\
+(\mu-\alpha \varepsilon) G
\end{array}\right]-\frac{\eta_{m} E^{2}}{2}\right\}-\frac{\eta_{r} A^{2}}{2}\right] d t \\
& \text { s.t. } \dot{T}(t)=\gamma E(t)-\delta T(t), T(0)=T_{0} \geq 0 \\
& \dot{G}(t)=\phi T(t)+\lambda A(t)-\vartheta G(t), G(0)=G_{0} \geq 0
\end{aligned}
$$

Theorem 3 (1) The manufacturer's and retailer's optimal emission reduction decisions are as follows: 


$$
\left\{\begin{array}{l}
E^{a^{*}}=\frac{\gamma\left(\tau_{m} \pi_{2}+\pi_{1}\right)[\phi(\mu-\alpha \varepsilon)+\alpha(\rho+\vartheta)]}{\eta_{m}(\rho+\vartheta)(\rho+\delta)} \\
A^{a^{*}}=\frac{\left(\tau_{r} \pi_{1}+\pi_{2}\right)[k(\rho+\vartheta)+\lambda(\mu-\alpha \varepsilon)]}{\eta_{r}(\rho+\vartheta)}
\end{array}\right.
$$

(2) The optimal evolution path of low-carbon level is as follows:

Where $T_{\infty}^{a}=\frac{\gamma^{2}\left(\tau_{m} \pi_{2}+\pi_{1}\right)[\phi(\mu-\alpha \varepsilon)+\alpha(\rho+\vartheta)]}{\delta \eta_{m}(\rho+\vartheta)(\rho+\delta)}$.

(3) The optimal evolution paths of low-carbon goodwill and reference low carbon level are as follows: :

$$
T^{a}(t)=\left(T_{0}-T_{\infty}^{a}\right) e^{-\delta t}+T_{\infty}^{a}
$$

$$
\begin{gathered}
G^{a}(t)=\left(G_{0}+B^{a}-G_{\infty}^{a}\right) e^{-\vartheta t}-B^{a} e^{-\delta t}+G_{\infty}^{a} \\
R^{a}(t)=\varepsilon G^{a}(t)
\end{gathered}
$$

Where $B^{a}=\frac{\phi T_{0}}{\delta-\vartheta}-\frac{\phi \gamma^{2}\left(\tau_{m} \pi_{2}+\pi_{1}\right)[\phi(\mu-\alpha \varepsilon)+\alpha(\rho+\vartheta)]}{\delta \eta_{m}(\rho+\vartheta)(\rho+\delta)(\delta-\vartheta)}$,

$$
G_{\infty}^{a}=\frac{\phi \gamma^{2}\left(\tau_{m} \pi_{2}+\pi_{1}\right)[\phi(\mu-\alpha \varepsilon)+\alpha(\rho+\vartheta)]}{\delta \vartheta \eta_{m}(\rho+\vartheta)(\rho+\delta)}+\frac{\lambda\left(\tau_{r} \pi_{1}+\pi_{2}\right)[k(\rho+\vartheta)+\lambda(\mu-\alpha \varepsilon)]}{\vartheta \eta_{r}(\rho+\vartheta)} .
$$

(4) The optimal profits of the manufacturer and retailer are as follows:

$$
\begin{aligned}
& \pi_{m}^{a}(t)=\pi_{m \infty}^{a}+a_{5}^{*}\left(T_{0}-T_{\infty}^{a}\right) e^{-\delta t}+b_{5}^{*}\left[\left(G_{0}+B^{a}-G_{\infty}^{a}\right) e^{-\vartheta t}-B^{a} e^{-\delta t}\right] \\
& \pi_{r}^{a}(t)=\pi_{r \infty}^{a}+a_{4}^{*}\left(T_{0}-T_{\infty}^{a}\right) e^{-\delta t}+b_{4}^{*}\left[\left(G_{0}+B^{a}-G_{\infty}^{a}\right) e^{-\vartheta t}-B^{a} e^{-\delta t}\right]
\end{aligned}
$$

(5) The altruistic utilities of the manufacturer and retailer are as follows:

$$
\begin{aligned}
& U_{m}^{a}(t)=\pi_{m \infty}^{a}+\tau_{m} \pi_{r \infty}^{a}+\left(a_{5}^{*}+\tau_{m} a_{4}^{*}\right)\left(T_{0}-T_{\infty}^{a}\right) e^{-\delta t}+\left(b_{5}^{*}+\tau_{m} b_{4}^{*}\right)\left[\left(G_{0}+B^{a}-G_{\infty}^{a}\right) e^{-\vartheta t}-B^{a} e^{-\delta t}\right] \\
& U_{r}^{a}(t)=\pi_{r \infty}^{a}+\tau_{r} \pi_{m \infty}^{a}+\left(a_{4}^{*}+\tau_{r} a_{5}^{*}\right)\left(T_{0}-T_{\infty}^{a}\right) e^{-\delta t}+\left(b_{4}^{*}+\tau_{r} b_{5}^{*}\right)\left[\left(G_{0}+B^{a}-G_{\infty}^{a}\right) e^{-\vartheta t}-B^{a} e^{-\delta t}\right]
\end{aligned}
$$

Where $\pi_{m \infty}^{a}=a_{5}^{*} T_{\infty}^{a}+b_{5}^{*} G_{\infty}^{a}+c_{5}^{*}, \pi_{r \infty}^{a}=a_{4}^{*} T_{\infty}^{a}+b_{4}^{*} G_{\infty}^{a}+c_{4}^{*}, a_{4}^{*}=\frac{\pi_{2}[\phi(\mu-\alpha \varepsilon)+\alpha(\rho+\vartheta)]}{(\rho+\vartheta)(\rho+\delta)}$

$$
a_{5}^{*}=\frac{\pi_{1}[\phi(\mu-\alpha \varepsilon)+\alpha(\rho+\vartheta)]}{(\rho+\vartheta)(\rho+\delta)} \quad, \quad b_{4}^{*}=\frac{\pi_{2}(\mu-\alpha \varepsilon)}{\rho+\vartheta} \quad, \quad b_{5}^{*}=\frac{\pi_{1}(\mu-\alpha \varepsilon)}{\rho+\vartheta}
$$

$$
c_{5}^{*}=\frac{1}{\rho}\left\{\pi_{1} v+\frac{\gamma^{2}\left[\pi_{1}^{2}-\left(\tau_{m} \pi_{2}\right)^{2}\right][\phi(\mu-\alpha \varepsilon)+\alpha(\rho+\vartheta)]^{2}}{2 \eta_{m}(\rho+\vartheta)^{2}(\rho+\delta)^{2}}+\frac{\left(\tau_{r} \pi_{1}+\pi_{2}\right)^{2}\left(1-\pi_{2}+\tau_{r} \pi_{1}\right)[(\rho+\vartheta) k+\lambda(\mu-\alpha \varepsilon)]^{2}}{2 \eta_{r} \tau_{r}(\rho+\vartheta)^{2}}\right\}
$$


$c_{4}^{*}=\frac{1}{\rho}\left\{\pi_{2} v+\frac{\gamma^{2}\left(\tau_{m} \pi_{2}+\pi_{1}\right) \pi_{2}[\phi(\mu-\alpha \varepsilon)+\alpha(\rho+\vartheta)]^{2}}{\eta_{m}(\rho+\vartheta)^{2}(\rho+\delta)^{2}}+\frac{\left[\pi_{2}^{2}-\left(\tau_{r} \pi_{1}\right)^{2}\right]\left(\tau_{r} \pi_{1}+\pi_{2}\right)[(\rho+\vartheta) k+\lambda(\mu-\alpha \varepsilon)]^{2}}{2 \eta_{r}(\rho+\vartheta)^{2}}\right\}$

389

\section{Proof (See Appendix).}

\subsection{Decentralized Scenario with Altruistic Behavior and a Cost-Sharing Contract}

At this time, the retailer is willing to share a certain percentage of emission reduction cost $\xi$ for the manufacturer. Similarly, the manufacturer's objective function is:

$J_{m}^{y}(T, G, t)=\max _{E} \int_{0}^{\infty} e^{-\rho t}\left[\pi_{1}\left[\begin{array}{l}v+\alpha T+k A \\ +(\mu-\alpha \varepsilon) G\end{array}\right]+\tau_{m}\left\{\begin{array}{l}\pi_{2}[v+\alpha T+k A+(\mu-\alpha \varepsilon) G] \\ -\frac{\eta_{r} A^{2}}{2}-\frac{\eta_{m} \xi E^{2}}{2}\end{array}\right\}-\frac{\eta_{m}(1-\xi) E^{2}}{2}\right] d t$

s.t. $\dot{T}(t)=\gamma E(t)-\delta T(t), T(0)=T_{0} \geq 0$

$$
\dot{G}(t)=\phi T(t)+\lambda A(t)-\vartheta G(t), G(0)=G_{0} \geq 0
$$

The retailer's objective function is:

$J_{r}^{y}(T, G, t)=\max _{A} \int_{0}^{\infty} e^{-\rho t}\left[\pi_{2}\left[\begin{array}{l}v+\alpha T+k A \\ +(\mu-\alpha \varepsilon) G\end{array}\right]+\tau_{r}\left\{\begin{array}{l}\pi_{1}[v+\alpha T+k A+(\mu-\alpha \varepsilon) G] \\ -\frac{\eta_{m}(1-\xi) E^{2}}{2}\end{array}\right\}-\frac{\eta_{r} A^{2}}{2}-\frac{\eta_{m} \xi E^{2}}{2}\right] d t$

s.t. $T(t)=\gamma E(t)-\delta T(t), T(0)=T_{0} \geq 0$

$\dot{G}(t)=\phi T(t)+\lambda A(t)-\vartheta G(t), G(0)=G_{0} \geq 0$

Theorem 4 (1) The manufacturer's and retailer's optimal emission reduction decisions are as follows:

$$
\left\{\begin{array}{c}
E^{y^{*}}=\frac{\gamma\left(\tau_{m} \pi_{2}+\pi_{1}\right)[\phi(\mu-\alpha \varepsilon)+\alpha(\rho+\vartheta)]}{\eta_{m}(\rho+\vartheta)(\rho+\delta)\left[1-\left(1-\tau_{m}\right) \xi\right]} \\
A^{y^{*}}=\frac{\left(\tau_{r} \pi_{1}+\pi_{2}\right)[k(\rho+\vartheta)+\lambda(\mu-\alpha \varepsilon)]}{\eta_{r}(\rho+\vartheta)}
\end{array}\right.
$$

(2) The optimal evolution path of low-carbon level is as follows:

$$
T^{y}(t)=\left(T_{0}-T_{\infty}^{y}\right) e^{-\delta t}+T_{\infty}^{y}
$$

Where $T_{\infty}^{y}=\frac{\gamma^{2}\left(\tau_{m} \pi_{2}+\pi_{1}\right)[\phi(\mu-\alpha \varepsilon)+\alpha(\rho+\vartheta)]}{\delta \eta_{m}(\rho+\vartheta)(\rho+\delta)\left[1-\left(1-\tau_{m}\right) \xi\right]}$.

(3) The optimal evolution paths of low-carbon goodwill and reference low carbon level are as follows: :

$$
G^{y}(t)=\left(G_{0}+B^{y}-G_{\infty}^{y}\right) e^{-\vartheta t}-B^{y} e^{-\delta t}+G_{\infty}^{y}
$$

$$
R^{y}(t)=\varepsilon G^{y}(t)
$$

Where $B^{y}=\frac{\phi T_{0}}{\delta-\vartheta}-\frac{\phi \gamma^{2}\left(\tau_{m} \pi_{2}+\pi_{1}\right)[\phi(\mu-\alpha \varepsilon)+\alpha(\rho+\vartheta)]}{\delta \eta_{m}(\rho+\vartheta)(\rho+\delta)(\delta-\vartheta)\left[1-\left(1-\tau_{m}\right) \xi\right]}$ 


$$
G_{\infty}^{y}=\frac{\phi \gamma^{2}\left(\tau_{m} \pi_{2}+\pi_{1}\right)[\phi(\mu-\alpha \varepsilon)+\alpha(\rho+\vartheta)]}{\delta \vartheta \eta_{m}(\rho+\vartheta)(\rho+\delta)\left[1-\left(1-\tau_{m}\right) \xi\right]}+\frac{\lambda\left(\tau_{r} \pi_{1}+\pi_{2}\right)[k(\rho+\vartheta)+\lambda(\mu-\alpha \varepsilon)]}{\vartheta \eta_{r}(\rho+\vartheta)} .
$$

(4) The optimal profits of the manufacturer and retailer are as follows:

$$
\begin{aligned}
& \pi_{m}^{y}(t)=\pi_{m \infty}^{y}+a_{5}^{*}\left(T_{0}-T_{\infty}^{y}\right) e^{-\delta t}+b_{5}^{*}\left[\left(G_{0}+B^{y}-G_{\infty}^{y}\right) e^{-\vartheta t}-B^{y} e^{-\delta t}\right] \\
& \pi_{r}^{y}(t)=\pi_{r \infty}^{y}+a_{4}^{*}\left(T_{0}-T_{\infty}^{y}\right) e^{-\delta t}+b_{4}^{*}\left[\left(G_{0}+B^{y}-G_{\infty}^{y}\right) e^{-\vartheta t}-B^{y} e^{-\delta t}\right]
\end{aligned}
$$

(5) The altruistic utilities of the manufacturer and retailer are as follows:

$$
\begin{aligned}
& U_{m}^{y}(t)=\pi_{m \infty}^{y}+\tau_{m} \pi_{r \infty}^{y}+\left(a_{5}^{*}+\tau_{m} a_{4}^{*}\right)\left(T_{0}-T_{\infty}^{y}\right) e^{-\delta t}+\left(b_{5}^{*}+\tau_{m} b_{4}^{*}\right)\left[\left(G_{0}+B^{y}-G_{\infty}^{y}\right) e^{-\vartheta t}-B^{y} e^{-\delta t}\right] \\
& U_{r}^{y}(t)=\tau_{r} \pi_{m \infty}^{y}+\pi_{r \infty}^{y}+\left(\tau_{r} a_{5}^{*}+a_{4}^{*}\right)\left(T_{0}-T_{\infty}^{y}\right) e^{-\delta t}+\left(\tau_{r} b_{5}^{*}+b_{4}^{*}\right)\left[\left(G_{0}+B^{y}-G_{\infty}^{y}\right) e^{-\vartheta t}-B^{y} e^{-\delta t}\right]
\end{aligned}
$$

Where $\pi_{m \infty}^{y}=a_{5}^{*} T_{\infty}^{y}+b_{5}^{*} G_{\infty}^{y}+c_{7}^{*}, \pi_{r \infty}^{y}=a_{4}^{*} T_{\infty}^{y}+b_{4}^{*} G_{\infty}^{y}+c_{6}^{*}, a_{4}^{*}=\frac{\pi_{2}[\phi(\mu-\alpha \varepsilon)+\alpha(\rho+\vartheta)]}{(\rho+\vartheta)(\rho+\delta)}$, $a_{5}^{*}=\frac{\pi_{1}[\phi(\mu-\alpha \varepsilon)+\alpha(\rho+\vartheta)]}{(\rho+\vartheta)(\rho+\delta)}, b_{4}^{*}=\frac{\pi_{2}(\mu-\alpha \varepsilon)}{\rho+\vartheta}, b_{5}^{*}=\frac{\pi_{1}(\mu-\alpha \varepsilon)}{\rho+\vartheta}$,

$c_{6}^{*}=\frac{1}{\rho}\left\{\begin{array}{l}\pi_{2} v+\frac{\gamma^{2}\left(\tau_{m} \pi_{2}+\pi_{1}\right)\left(2 \pi_{2}-2 \pi_{2} \xi+\tau_{m} \pi_{2} \xi-\pi_{1} \xi\right)[\phi(\mu-\alpha \varepsilon)+\alpha(\rho+\vartheta)]^{2}}{2 \eta_{m}(\rho+\vartheta)^{2}(\rho+\delta)^{2}\left[1-\left(1-\tau_{m}\right) \xi\right]^{2}} \\ +\frac{\left[\pi_{2}^{2}-\left(\tau_{r} \pi_{1}\right)^{2}\right][(\rho+\vartheta) k+\lambda(\mu-\alpha \varepsilon)]^{2}}{2 \eta_{r}(\rho+\vartheta)^{2}}\end{array}\right\}$

$c_{7}^{*}=\frac{1}{\rho}\left\{\begin{array}{l}\pi_{1} v+\frac{\gamma^{2}\left(\tau_{m} \pi_{2}+\pi_{1}\right)\left[(1-\xi)\left(\pi_{1}-\tau_{m} \pi_{2}\right)+2 \tau_{m} \pi_{1} \xi\right][\phi(\mu-\alpha \varepsilon)+\alpha(\rho+\vartheta)]^{2}}{2 \eta_{m}(\rho+\vartheta)^{2}(\rho+\delta)^{2}\left[1-\left(1-\tau_{m}\right) \xi\right]^{2}} \\ +\frac{\left(\tau_{r} \pi_{1}+\pi_{2}\right) \pi_{1}[(\rho+\vartheta) k+\lambda(\mu-\alpha \varepsilon)]^{2}}{\eta_{r}(\rho+\vartheta)^{2}}\end{array}\right\}$.

The proof of Theorem 4 is similar to the proof of Theorem 3 and is omitted.

Theorem 5 (1) The optimal emission reduction cost sharing ratio provided by the retailer is as follows:

$$
\xi^{*}= \begin{cases}\frac{2\left(1-\tau_{m}\right)\left[\left(\tau_{r} \pi_{1}+\pi_{2}\right)-\left(\tau_{m} \pi_{2}+\pi_{1}\right)\right]-\left(1-\tau_{r}\right)\left(\tau_{m} \pi_{2}+\pi_{1}\right)}{\left(1-\tau_{m}\right)\left[2\left(1-\tau_{m}\right)\left(\tau_{r} \pi_{1}+\pi_{2}\right)+\left(1-\tau_{r}\right)\left(\tau_{m} \pi_{2}+\pi_{1}\right)\right]}, & 2 A \geq B \\ 0, & 2 A<B\end{cases}
$$

Where $A=\left(1-\tau_{m}\right)\left[\left(\tau_{r} \pi_{1}+\pi_{2}\right)-\left(\tau_{m} \pi_{2}+\pi_{1}\right)\right], B=\left(1-\tau_{r}\right)\left(\tau_{m} \pi_{2}+\pi_{1}\right)$.

(2) When the retailer is totally altruistic $\left(\tau_{r}=1\right)$, there is $\xi^{*}=\frac{\pi_{2}}{\pi_{1}+\pi_{2}}, E^{y^{*}}=E^{c^{*}}, A^{y^{*}}=A^{c^{*}}$, $\pi_{s c}^{c}(t)=\pi_{m}^{y}(t)+\pi_{r}^{y}(t)$, this cost-sharing contract can achieve perfect coordination of the supply chain. Theorem 5 is easy to proof and is omitted. 
Corollary 1 The relationship between related parameters under decentralized decision scenario with altruistic behavior is shown in the Table 3.

Table 3. The relationship between related parameters

\begin{tabular}{lllllll}
\hline & $E$ & $A$ & $G_{\infty}$ & $R_{\infty}$ & $T_{\infty}$ & $D_{\infty}$ \\
\hline$\pi_{1}$ & + & + & + & + & + & + \\
$\pi_{2}$ & + & + & + & + & + & + \\
$\tau_{m}$ & + & $\times$ & + & + & + & + \\
$\tau_{r}$ & $\times$ & + & + & + & + & + \\
$\eta_{m}$ & - & $\times$ & - & - & - & - \\
$\eta_{r}$ & $\times$ & - & - & - & + & - \\
$\alpha$ & + & + & + & + & + & + \\
$k$ & $\times$ & $\times$ & + & + & $\times$ & + \\
$\phi$ & + & - & - & + & + & + \\
$\varepsilon$ & + & $\times$ & + & + & + & - \\
$\gamma$ & + & + & + & + & + & + \\
$\lambda$ & + & + & + & + & + & + \\
$\mu$ & - & - & - & - & + & + \\
$\rho$ & + & + & + & + & + \\
\hline
\end{tabular}

Note: "+" denotes positive correlation; "-" denotes negative correlation; " $\times$ " denotes irrelevant; " \pm " denotes positive or negative correlation depends on the situation.

Corollary 1 shows:(1) As the marginal revenue of manufacturers and retailers continues to increase, manufacturers' investment in emission reduction and retailers' investment in low-carbon publicity will also continue to increase, which will positively promote the low-carbon goodwill, further stimulate consumer demand, increase product market demand and finally bring good benefits to the supply chain. Therefore, when the supply chain members' altruism intensity is certain, in order to further highlight the positive effects of altruistic behavior, the supply chain members should start from their own point of view and increase their own marginal profits through a variety of ways, thereby enhancing the positive impact of supply chain members' altruistic behavior on the entire supply chain.

(2) As both parties' altruism intensity continues to increase, manufacturers' investment in emission reduction and retailers' investment in low-carbon publicity will continue to increase, which will promote the increase in low-carbon level, low-carbon goodwill and market demand. This means that, within the reasonable scope of their own altruism intensity, supply chain members showing a higher altruistic intensity to the other party can effectively promote the cooperation between the two parties, which is not only conducive to improving their own benefits, but also bring products with higher low-carbon level and low-carbon goodwill to consumers, thereby further enhance the market competitive advantage of the overall supply chain.

(3) With the increasing impact of the reference low-carbon effect on product demand, retailers' low-carbon publicity investment will continue to decrease, while manufacturers' investment in emission reduction and product low-carbon level will continue to increase, low-carbon goodwill and market demand will not change significantly. This shows that when the reference low-carbon effect has a significant impact on the market demand, supply chain members will adjust their measures accordingly. The specific manifestation is that they will pay more attention to the low-carbon level of products, so manufacturers will increase investment in emission reduction to improve the low-carbon level of products. At the same time, since product low-carbon goodwill and market demand are jointly 
affected by product low-carbon level and retailers' low-carbon publicity investment, there is no significant change between product low-carbon goodwill and market demand at this time.

(4) As the relevance degree between the reference low-carbon level and product low-carbon goodwill continues to increase, manufacturers' investment in emission reduction and retailers' investment in low-carbon publicity will continue to decline, and the low-carbon goodwill will also continue to decline. This means that consumers ignorantly judging the low-carbon level of products based on the low-carbon goodwill level will not only bring losses to the overall profit of the supply chain, but also adversely affect the low-carbon level of products they obtain.

(5) As the discount rate continues to increase, manufacturers' investment in emission reduction and retailers' investment in low-carbon publicity will continue to decline, resulting in a decline in product low-carbon level, low-carbon goodwill and ultimately a reduction in product market demand. The higher the discount rate, the more the company cares about short-term profits. At this time, the manufacturer's emission reduction investment and the retailer's low-carbon publicity investment are difficult to be perceived by the market in a short time, so the higher discount at this time will inhibit the enthusiasm of manufacturers and retailers to invest in emissions reduction.

Corollary 2 By comparing manufacturers' emission reduction investment, retailers' low-carbon publicity investment, steady-state low-carbon level, low-carbon goodwill and product demand under four decision scenarios, we can obtain: $E^{c} \geq E^{y} \geq E^{a} \geq E^{d}, A^{c} \geq A^{y}=A^{a} \geq A^{d}, T_{\infty}^{c} \geq T_{\infty}^{y} \geq T_{\infty}^{a} \geq T_{\infty}^{d}$,

$G_{\infty}^{c} \geq G_{\infty}^{y} \geq G_{\infty}^{a} \geq G_{\infty}^{d}, Q_{\infty}^{c} \geq Q_{\infty}^{y} \geq Q_{\infty}^{a} \geq Q_{\infty}^{d}$

It can be seen from Corollary 2 that under centralized decision scenario, the manufacturer's emission reduction investment and the retailer's low-carbon publicity investment are both the largest. The altruistic behavior of the manufacturer and the retailer can effectively promote both parties to increase emission reduction investment, make the emission reduction investment of both parties no less than that when they are fully self-interested, but not higher than the emission reduction investment of both parties under centralized decision scenario. In particular, when both parties are completely altruistic $\left(\tau_{m}=\tau_{r}=1\right)$, both parties' emission reduction investment can reach the level of centralized decision scenario. Retailers sharing emission reduction costs for manufacturers can effectively encourage manufacturers to increase emission reduction investment, but the increased emission reduction investment of manufacturers still cannot reach the level of centralized decision scenario. At this time, the retailer's low-carbon publicity investment is not lower than the low-carbon publicity investment when there is no cost sharing, but it cannot reach the low-carbon publicity investment level under centralized decision scenario.

Corollary 3 Comparing the evolution path of product low-carbon level under four decision scenarios, we can obtain:

$$
T^{c}(t) \geq T^{y}(t) \geq T^{a}(t) \geq T^{d}(t) \quad, \quad \frac{\partial\left(T_{\infty}^{c}-T_{\infty}^{y}\right)}{\partial \tau_{m}}=\frac{\partial\left(T^{c}(t)-T^{y}(t)\right)}{\partial \tau_{m}}<0
$$

$\frac{\partial\left(T_{\infty}^{c}-T_{\infty}^{a}\right)}{\partial \tau_{m}}=\frac{\partial\left(T^{c}(t)-T^{a}(t)\right)}{\partial \tau_{m}}<0$

;when

$T_{0}<T_{\infty}^{d}<T_{\infty}^{a}<T_{\infty}^{y}$

,there is

$\frac{\partial\left(T^{y}(t)\right)}{\partial t}>\frac{\partial\left(T^{a}(t)\right)}{\partial t}>\frac{\partial\left(T^{d}(t)\right)}{\partial t}>0$

,when

$T_{0}>T_{\infty}^{y}>T_{\infty}^{a}>T_{\infty}^{d} \quad$,there 
$\frac{\partial\left(T^{d}(t)\right)}{\partial t}<\frac{\partial\left(T^{a}(t)\right)}{\partial t}<\frac{\partial\left(T^{y}(t)\right)}{\partial t}<0$; when $T_{0}=T_{\infty}^{d}$, there is $\frac{\partial\left(T^{d}(t)\right)}{\partial t}=0$, when $T_{0}=T_{\infty}^{a}$, there is $\frac{\partial\left(T^{a}(t)\right)}{\partial t}=0$, when $T_{0}=T_{\infty}^{y}$, there is $\frac{\partial\left(T^{y}(t)\right)}{\partial t}=0$.

It can be seen from Corollary 3 that the low-carbon level in centralized decision scenario is the highest, which is consistent with the conclusion that the manufacturer's investment in emission reduction is the largest under centralized decision scenario in Corollary 2. The altruistic behavior of the manufacturer can effectively improve the low-carbon level, and the emission reduction cost-sharing contract provided by the retailer can further enhance the low carbon level. In addition, as the intensity of the altruistic behavior of manufacturers continues to increase, the difference between the low-carbon level under centralized decision scenario and the low-carbon level under two altruistic decentralized decision scenarios will continue to shrink, which again verifies that the altruistic behavior of the manufacturer can effectively promote its increase in emissions reduction investment. If the initial low-carbon level of the product is less than the steady-state low-carbon level under each decentralized decision scenario, the low-carbon level of the product will continue to rise over time, otherwise, the low-carbon level of the product will continue to decline over time.

Corollary 4 Comparing the evolution trajectory of product low-carbon goodwill under four decision scenarios, we can obtain:

$$
G^{c}(t) \geq G^{y}(t) \geq G^{a}(t) \geq G^{d}(t) \quad, \quad \frac{\partial\left(G_{\infty}^{c}-G_{\infty}^{y}\right)}{\partial \tau_{m}}=\frac{\partial\left(G^{c}(t)-G^{y}(t)\right)}{\partial \tau_{m}}<0
$$

$$
\frac{\partial\left(G_{\infty}^{c}-G_{\infty}^{y}\right)}{\partial \tau_{r}}=\frac{\partial\left(G^{c}(t)-G^{y}(t)\right)}{\partial \tau_{r}}<0 \quad, \quad \frac{\partial\left(G_{\infty}^{c}-G_{\infty}^{a}\right)}{\partial \tau_{m}}=\frac{\partial\left(G^{c}(t)-G^{a}(t)\right)}{\partial \tau_{m}}<0
$$

$\frac{\partial\left(G_{\infty}^{c}-G_{\infty}^{a}\right)}{\partial \tau_{r}}=\frac{\partial\left(G^{c}(t)-G^{a}(t)\right)}{\partial \tau_{r}}<0$

,when

$$
G_{0}<G_{\infty}^{d}<G_{\infty}^{a}<G_{\infty}^{y}
$$

$$
\frac{\partial\left(G^{y}(t)\right)}{\partial t}>\frac{\partial\left(G^{a}(t)\right)}{\partial t}>\frac{\partial\left(G^{d}(t)\right)}{\partial t}>0
$$

$\frac{\partial\left(G^{d}(t)\right)}{\partial t}<\frac{\partial\left(G^{a}(t)\right)}{\partial t}<\frac{\partial\left(G^{y}(t)\right)}{\partial t}<0$; when $G_{0}=G_{\infty}^{d}$, there is $\frac{\partial\left(G^{d}(t)\right)}{\partial t}=0$, when $G_{0}=G_{\infty}^{a}$, there is

$$
\frac{\partial\left(G^{a}(t)\right)}{\partial t}=0, \text { when } G_{0}=G_{\infty}^{y}, \text { there is } \frac{\partial\left(G^{y}(t)\right)}{\partial t}=0 \text {. }
$$

It can be seen from Corollary 4 that similar to low-carbon level, the low-carbon goodwill in centralized decision scenario is the highest, which is consistent with the conclusion that the manufacturer's investment in emission reduction and the retailer's investment in low-carbon publicity are both the largest under centralized decision scenario in Corollary 2. Both parties' altruistic behavior can effectively improve the low-carbon goodwill, and the emission reduction cost-sharing contract provided by the retailer can further enhance the low carbon goodwill. In addition, as the intensity of the altruistic behavior of both parties continues to increase, the difference between the low-carbon 
decentralized decision scenarios will continue to shrink, which again verifies that the altruistic behavior of the manufacturer can effectively promote its increase in emissions reduction investment and the altruistic behavior of the retailer can effectively promote its increase in low-carbon publicity investment. If the initial low-carbon goodwill of the product is less than the steady-state low-carbon goodwill under each decentralized decision scenario, the low-carbon goodwill of the product will continue to rise over time, otherwise, the low-carbon goodwill of the product will continue to decline over time.

Corollary 5 Under decentralized decision scenario with altruistic behavior and a cost-sharing contract, we can obtain: $\frac{\partial E^{y^{*}}}{\partial \xi}>0, \frac{\partial \xi}{\partial \tau_{m}}<0, \frac{\partial \xi}{\partial \tau_{r}}>0, \frac{\partial \xi}{\partial \pi_{1}}<0, \frac{\partial \xi}{\partial \pi_{2}}>0 ; \frac{\partial \pi_{r \infty}^{y}}{\partial \tau_{m}} \geq 0, \frac{\partial \pi_{m \infty}^{y}}{\partial \tau_{m}}<0$, $\frac{\partial U_{m}^{y}(t)}{\partial \tau_{m}} \geq 0, \frac{\partial U_{m}^{y}(t)}{\partial \tau_{r}} \geq 0, \frac{\partial U_{r}^{y}(t)}{\partial \tau_{m}} \geq 0, \frac{\partial U_{r}^{y}(t)}{\partial \tau_{r}} \geq 0$.

It can be seen from Corollary 5 that the greater the sharing ratio of emission reduction costs provided by the retailer to the manufacturer, the greater the incentive for the manufacturer to invest in emission reductions. The sharing ratio of emission reduction costs provided by the retailer to the manufacturer will decrease with the increase in the intensity of the manufacturer's altruistic behavior and the manufacturer's marginal revenue, and increase with the increase in the intensity of the retailer's altruistic behavior and the retailer's marginal revenue. This is consistent with the reality. When retailers pay more attention to manufacturers' profits and their marginal revenue increases, they will naturally strengthen their incentives for manufacturers to invest in emission reductions. On the contrary, when manufacturers pay more attention to retailers' profits and their marginal revenue increases, it is not difficult to understand that at this time manufacturers have a strong economic power to invest in emission reductions, so the retailer will reduce its economic support for the manufacturer's emission reduction investment. In addition, the manufacturer's profit will decrease as its intensity of the altruistic behavior increases, and the retailer's profit will increase as the manufacturer's intensity of the altruistic behavior increases. Furthermore, as the intensity of the altruistic behavior of both parties continues to increase, both parties' utilities will continue to increase.

Corollary 6 Under decentralized decision scenario with altruistic behavior, we can obtain:

when $0 \leq \tau_{m} \leq \frac{\rho \vartheta \pi_{1}[\phi(\mu-\alpha \varepsilon)+\alpha(\rho+\vartheta)]+\rho \phi \pi_{1}(\rho+\delta)(\mu-\alpha \varepsilon)}{\pi_{2} \delta \vartheta[\phi(\mu-\alpha \varepsilon)+\alpha(\rho+\vartheta)]}$, then $\frac{\partial \pi_{m \infty}^{a}}{\partial \tau_{m}} \geq 0$,conversely, $\frac{\partial \pi_{m \infty}^{a}}{\partial \tau_{m}}<0 ; \frac{\partial \pi_{m \infty}^{a}}{\partial \tau_{r}}>0 ; \frac{\partial \pi_{r \infty}^{a}}{\partial \tau_{m}}>0, \frac{\partial \pi_{r \infty}^{a}}{\partial \tau_{r}}<0 ; \frac{\partial U_{m}^{a}(t)}{\partial \tau_{m}} \geq 0, \frac{\partial U_{r}^{a}(t)}{\partial \tau_{m}} \geq 0, \frac{\partial U_{m}^{a}(t)}{\partial \tau_{r}} \geq 0, \frac{\partial U_{r}^{a}(t)}{\partial \tau_{r}} \geq 0$

It can be seen from Corollary 6 that the profits of the manufacturer and the retailer will increase with the increase in the other parties' altruistic intensity. Regardless of the retailer's altruistic intensity, the retailer's profit will decrease with the increase in the altruistic intensity, while the manufacturer's profit may increase or decrease with the increase in its altruistic intensity, depending on its altruistic intensity. In particular, when its altruistic intensity is lower than a certain value, the manufacturer's profit will increase as its altruistic intensity increases, otherwise it will decline. In addition, the utilities of both the manufacturer and the retailer will increase as the altruistic intensity of its own and the other party increases.

Corollary $7 \pi_{m}^{y}(t) \geq \pi_{m}^{a}(t) \geq \pi_{m}^{d}(t), \pi_{r}^{y}(t) \geq \pi_{r}^{a}(t) \geq \pi_{r}^{d}(t), U_{m}^{y}(t) \geq U_{m}^{a}(t), U_{r}^{y}(t) \geq U_{r}^{a}(t)$.

It can be seen from Corollary 7 that compared with when the two parties do not have altruistic 
behavior, the altruistic behavior of the manufacturer and the retailer can increase the profits of both parties. When the retailer chooses to share the emission reduction costs for the manufacturer, it can further improve the profit level of both parties. In addition, the emission reduction cost sharing contract provided by the retailer to the manufacturer can effectively improve the utilities of both parties, so the emission reduction cost-sharing contract can promote the coordination of the supply chain.

\section{Numerical Analysis}

To more intuitively reveal the impact of key parameters on the equilibrium solutions under different scenarios and obtain some valuable management enlightenments, we will conduct numerical analysis to further test the previous conclusions in this section. Based on the related researches and the specific research hypothesis of this paper, the parameters involved in this paper are set as follows: $\pi_{1}=6, \pi_{2}=5$ , $\eta_{m}=15, \eta_{r}=12, \rho=1, \varepsilon=0.3, \phi=1, \lambda=1, \alpha=1, \gamma=2, \delta=1, k=1, \mu=0.5, \vartheta=0.7, v=5$, $T_{0}=0, G_{0}=0, \tau_{m}=0.1, \tau_{r}=0.7$.

\subsection{Impact of Time}

(1) The optimal evolution paths of product low-carbon level, low-carbon goodwill, reference low-carbon level and demand over time under the four decision scenarios.

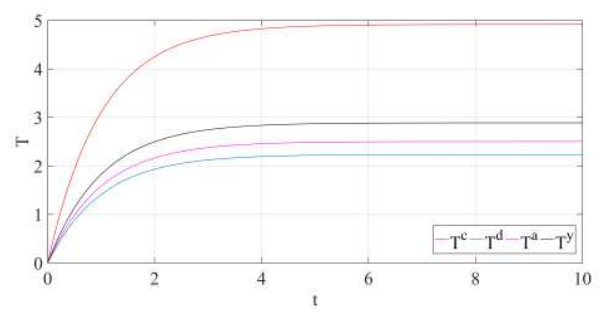

(a)

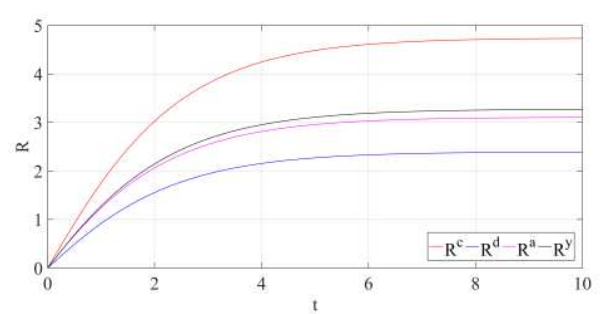

(c)

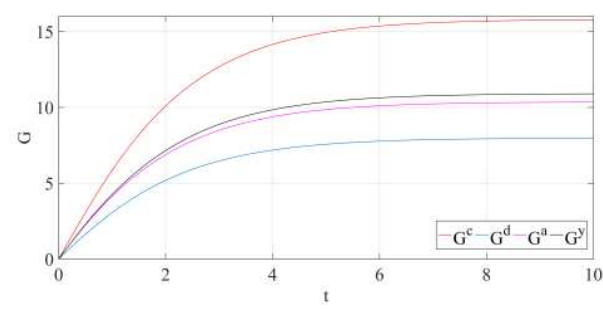

(b)

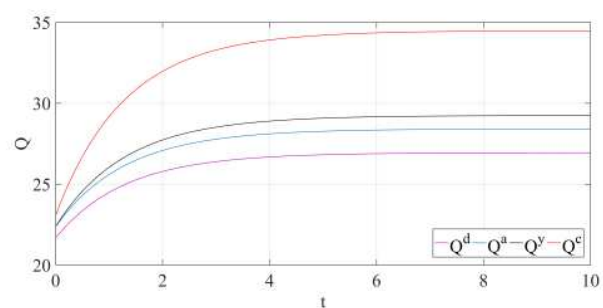

(d)

Figure 1. (a) The evolution path of low-carbon level $T(t)$.(b) The evolution path of low-carbon goodwill $G(t)$.(c) The evolution path of reference low-carbon level $R(t)$. (d) The evolution path of demand $Q(t)$.

It can be seen from Figure 1 that under the four decision scenarios, product low-carbon level, low-carbon goodwill, reference low-carbon level and product market demand all have a time-stabilizing trend, showing that they tend to a certain stable value when time tends to infinity. In addition, it can be seen that since the initial product low-carbon level and low-carbon goodwill are both assumed to be 0 at this time, which is less than the stable value, the product low-carbon level and low-carbon goodwill both show a monotonous increasing trend over time. At the same moment, each state quantity under centralized decision scenario is the highest, and it is much larger than that under each decentralized decision scenario. Each state quantity under decentralized decision scenario with altruistic behavior is 
higher than that under decentralized decision scenario without altruistic behavior. The above phenomenon shows that the altruistic behavior of manufacturers and retailers can help improve product low-carbon level and low-carbon goodwill, and when retailers share the cost of reducing emissions for manufacturers, it will help further improve product low-carbon level and low-carbon goodwill, and ultimately increase the market demand of low-carbon products.

(2) The optimal evolution paths of the manufacturer's profit, retailer's profit, overall supply chain profit, manufacturer's utility, retailer's utility and utility difference over time under different decision scenarios.

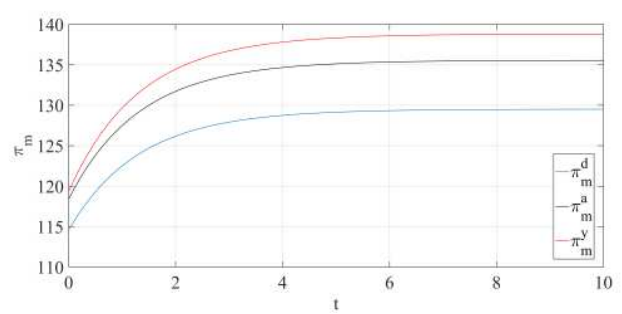

(a)

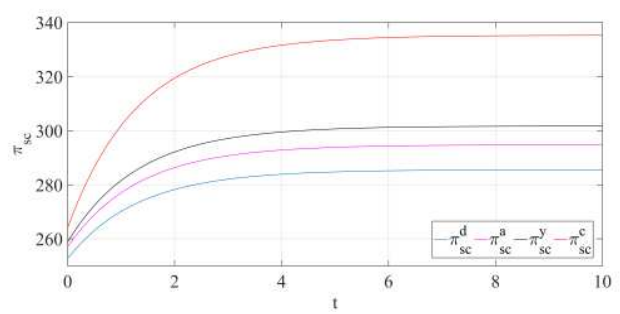

(c)

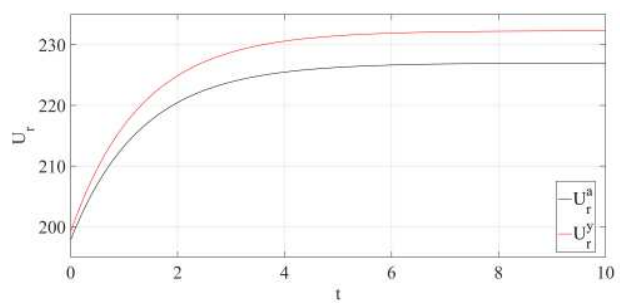

(e)

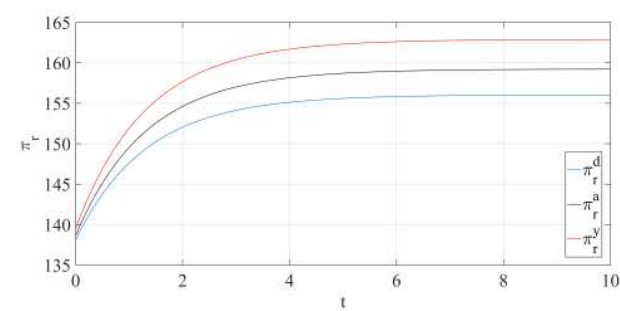

(b)

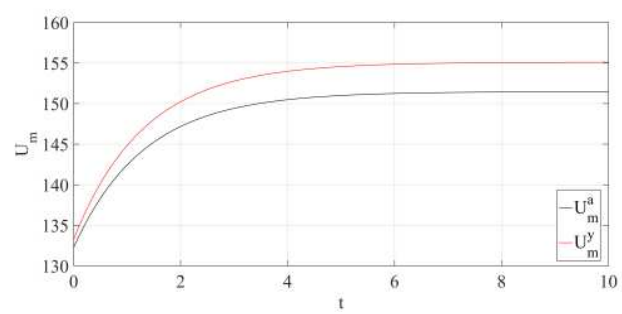

(d)

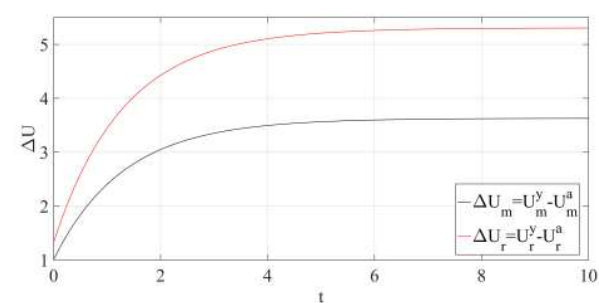

(f)

Figure 2. (a) The evolution path of the manufacturer's profit $\pi_{m}(t)$.(b) The evolution path of the retailer's profit $\pi_{r}(t)$.(c) The evolution path of overall supply chain profit $\pi_{s c}(t)$.(d) The evolution path of the manufacturer's utility $U_{m}(t)$.(e) The evolution path of the retailer's utility $U_{r}(t)$.(f) The evolution path of the utility difference $\Delta U(t)$.

It can be seen from Figure 2 (a) and (b) that as time goes by, the profits of the manufacturer, the retailer and the overall supply chain under each decision scenario continue to increase, and eventually tend to a certain stable value. Moreover, when both parties have altruistic behavior, the profits of both parties have been improved compared with when they do not have altruistic behavior. In addition, when retailers share emission reduction costs for manufacturers, the profit level of both parties has been further improved. From Figure 2(c), it can be seen that the overall profit of the supply chain under centralized decision scenario is much greater than that under each decentralized decision scenario. The overall profit of the supply chain under decentralized decision scenario with altruistic behavior and a cost-sharing contract is second. While the overall profit of the supply chain is the lowest under decentralized decision scenario without altruistic behavior. From Figure 2(d) and (e), it can be seen that both parties' utilities 
continue to increase over time, and eventually tend to a certain stable value. This shows that from a long-term perspective, if both the manufacturer and the retailer hold an altruistic attitude towards each other, it will not only contribute to the sustained and stable growth of the both parties' profits, but also produce lasting and stable social welfare. From Figure 2(f), it can be seen that the difference between the utilities of the manufacturer and the retailer under the two decision scenarios will increase over time, and eventually tends to a certain stable value. This means that when the manufacturer and the retailer both have altruistic behavior, if the retailer chooses to share the emission reduction costs for the manufacturer, the utilities of both parties will not only be further improved, but from a long-term perspective, both parties can obtain a sustained and stable utility increment, bringing lasting and stable social welfare.

(1) The impact of manufacturer's altruistic behavior on the manufacturer's profit, retailer's profit, manufacturer's utility and retailer's utility.

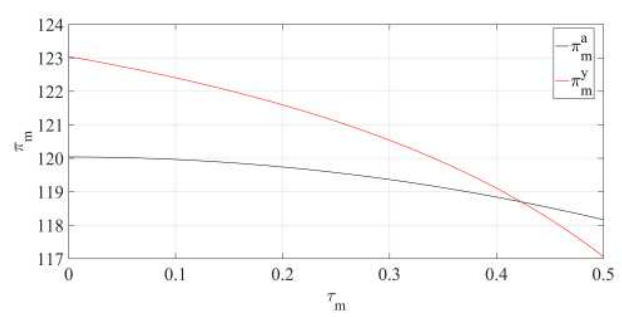

(a)

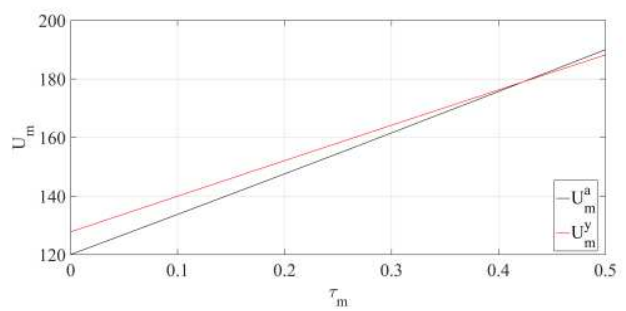

(c)

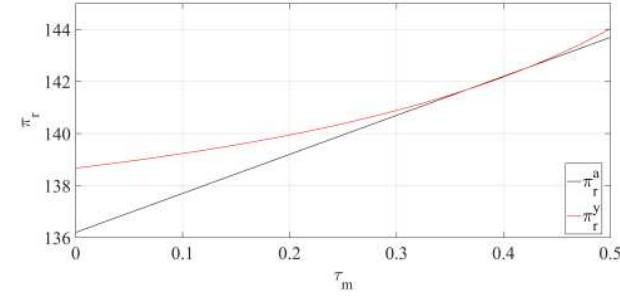

(b)

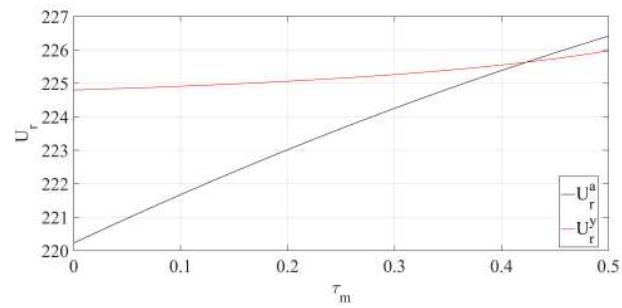

(d)

Figure 3. (a) The trajectory of the manufacturer's profit $\pi_{m}$.(b) The trajectory of the retailer's profit $\pi_{r}$ .(c) The trajectory of the manufacturer's utility $U_{m}$.(d) The trajectory of the retailer's utility $U_{r}$.

From Figure 3 (a) and (b), it can be seen that the profit of the manufacturer decreases as its altruistic intensity increases, while the profit of the retailer increases with the increase of the manufacturer's altruistic intensity. In addition, when the retailer chooses to share the emission reduction cost for the manufacturer, that is, when the manufacturer's altruistic intensity is less than a certain value at this time, the profit level of both parties has been further improved compared with when the emission reduction cost is not shared. From Figure 3(c) and (d), it can be seen that with the continuous increase in the manufacturer's altruistic intensity, the utilities of the manufacturer and the retailer under the two decision scenarios will both continue to increase, and when the retailer chooses to share the emission reduction cost for the manufacturer, that is, when the manufacturer's altruistic intensity is less than a certain value, the utility level of both parties has been further improved compared with when the emission reduction cost is not shared. In addition, it can be clearly seen that as the manufacturer's altruistic intensity continues to increase, the differences between the manufacturer's profit, the retailer's profit, the manufacturer's utility and the retailer's utility under the two decision scenarios will constantly decrease. The above phenomenon shows that if the manufacturer's altruistic intensity is high enough, 
which means that the manufacturer is very concerned about the retailer's profit, then compared with the retailer sharing the manufacturer's emission reduction cost, the manufacturer can gain more profits, greater utility, and the retailer can also obtain greater utility when the retailer does not share the manufacturer's emission reduction cost.

(2) The impact of retailer's altruistic behavior on the manufacturer's profit, retailer's profit, manufacturer's utility and retailer's utility.

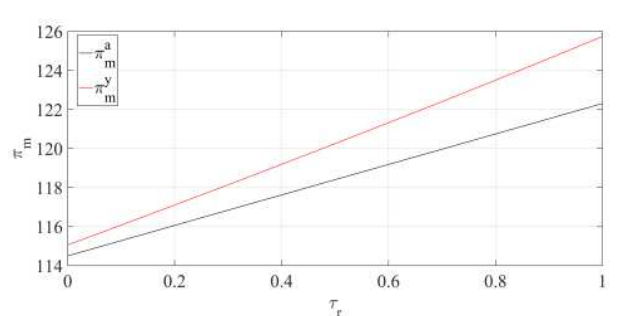

(a)

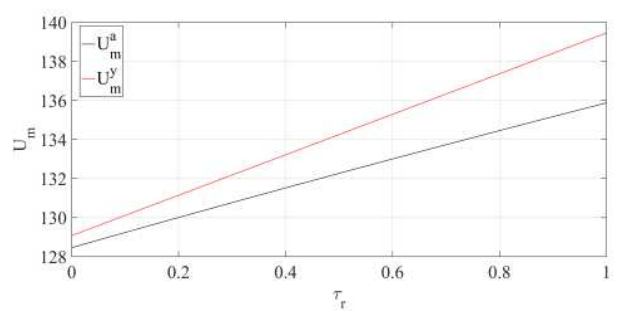

(c)

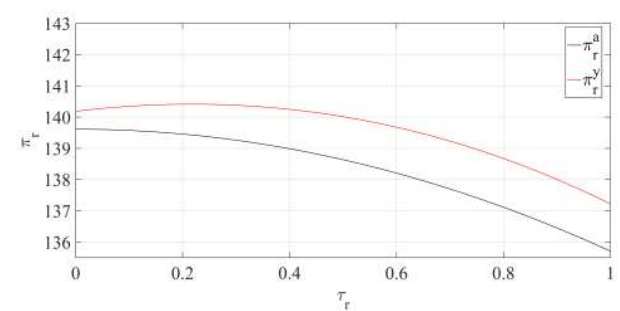

(b)

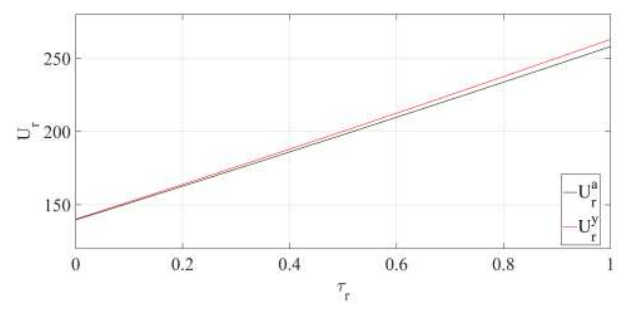

(d)

Figure 4. (a) The trajectory of the manufacturer's profit $\pi_{m}$.(b) The trajectory of the retailer's profit $\pi_{r}$ .(c) The trajectory of the manufacturer's utility $U_{m}$.(d) The trajectory of the retailer's utility $U_{r}$.

From Figure 4 (a) and (b), it can be seen that the profit of the manufacturer increases as the retailer's altruistic intensity increases, while the profit of the retailer decreases with the increase of its altruistic intensity. In addition, compared with when the manufacturer's emission reduction cost is not shared, when the retailer chooses to share the manufacturer's emission reduction cost, the profit level of both parties has been further improved. From Figure 4(c) and (d), it can be seen that with the increase of the retailer's altruism intensity, both parties' utilities under the two decision scenarios will continue to increase, and when the retailer chooses to share emission reduction cost for the manufacturer, the utility level of both parties has been further improved. In addition, it can be clearly seen that with the continuous increase of the retailer's altruistic intensity, the differences between the manufacturer's profit, the retailer's profit, the manufacturer's utility and the retailer's utility under the two decision scenarios will constantly increase. The above phenomenon shows that if the retailer's altruistic intensity is high enough, which means that the retailer pays much attention to the manufacturer's profit, then compared with the retailer not sharing the manufacturer's emission reduction cost, the manufacturer can gain more profits, greater utility, and retailers can also obtain greater profits and utility when the retailer shares the manufacturer's emission reduction cost.

According toTheorem 5 (2), the cost-sharing contract can achieve a win-win situation for supply chain members, because compared with no cost-sharing contract, both the manufacturer and the retailer can obtain a certain utility increment under the cost-sharing contract. Particularly, when the retailer is totally altruistic $\left(\tau_{r}=1\right)$, both the manufacturer's emission reduction investment and the retailer's low-carbon publicity investment can reach the investment levels under centralized decision scenario, 

when the retailer is totally altruistic $\left(\tau_{r}=1\right)$, the less the altruistic intensity of the manufacturer, the more the profit and utility increment the manufacturer and retailer can obtain.

(1) The impact of reference low-carbon effect on the manufacturer's emission reduction investment, the retailer's low-carbon publicity investment, steady-state low-carbon level and low-carbon goodwill.

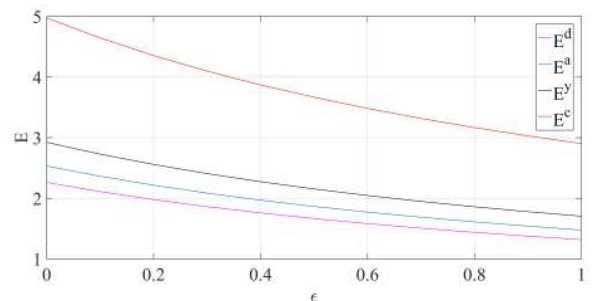

(a)

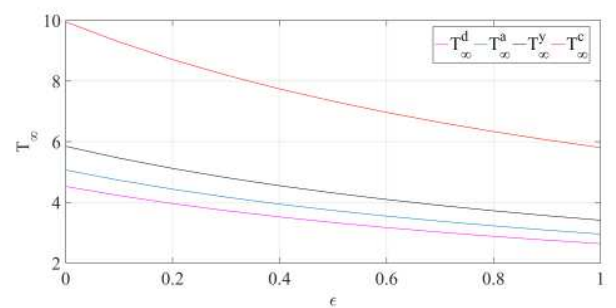

(c)

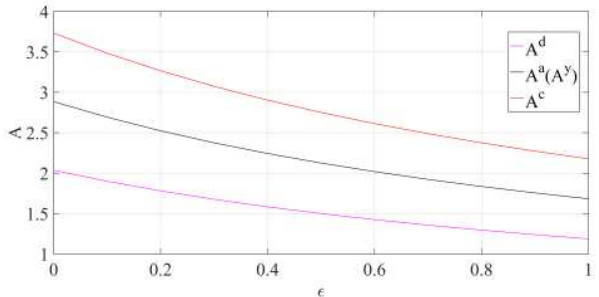

(b)

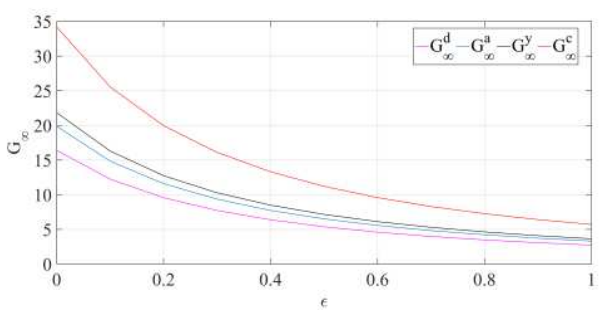

(d)

Figure 5. (a) The trajectory of the manufacturer's emission reduction investment $E$.(b) The trajectory of the retailer's low-carbon publicity investment $A$.(c) The trajectory of the steady-state low-carbon level $T_{\infty}$.(d) The trajectory of the steady-state low-carbon goodwill $G_{\infty}$.

It can be seen from Figure 5(a) that no matter what kind of decision scenarios, with the continuous increase of the reference low-carbon effect, the manufacturer's emission reduction investment will continue to decrease, but the reduction magnitude shows a marginal diminishing law. In addition, it can be clearly seen that compared with when both parties do not have altruistic behavior, the final stable value of the manufacturer's emission reduction investment is higher when both parties have altruistic behavior, and when the retailer shares the emission reduction costs for the manufacturer, the final stable value will be further improved. From Figure 5(b), it can be seen that under different decision scenarios, the retailer's low-carbon publicity investment also decreases with the continuous increase of the reference low-carbon effect, and the reduction magnitude also shows a marginal diminishing law. When both parties have altruistic behavior, the stable value of the retailer's low-carbon publicity investment is much higher. Directly affected by the manufacturer's investment in emission reduction and the retailer's low-carbon publicity investment, the steady-state low-carbon level and low-carbon goodwill also decrease as the reference low-carbon effect continues to increase, and the reduction magnitude also shows a marginal diminishing law. In particular, when consumers rely solely on low-carbon goodwill to determine the low-carbon level of the product $(\varepsilon=1)$, the low-carbon goodwill of the product will be reduced to a minimum level, but it will still be higher than the initial low-carbon goodwill level.

(2) The impact of reference low-carbon effect on the manufacturer's profit and the retailer's profit. 


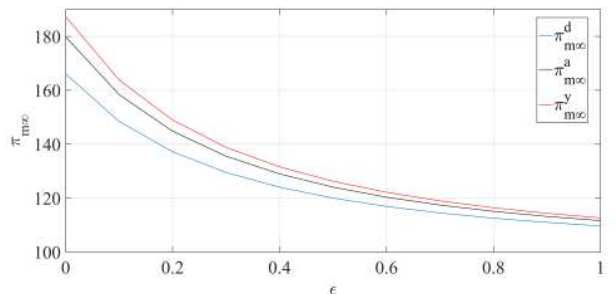

(a)

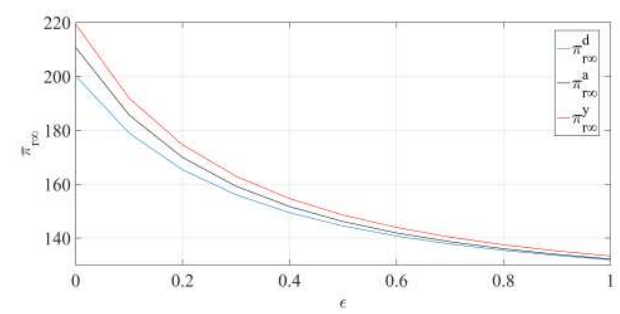

(b)

Figure 6. (a) The trajectory of the manufacturer's profit $\pi_{m}$.(b) The trajectory of the retailer's profit $\pi_{r}$

It can be seen from Figure 6(a) and (b) that no matter what kind of decision scenarios, with the continuous increase of the reference low-carbon effect, both parties' profits will continue to decrease, but the reduction magnitude shows a marginal diminishing law. When the reference low-carbon effect reaches its maximum ( $\varepsilon=1$ ), both parties' profits will stabilize at a minimum level. In addition, it can also be clearly seen that compared with when both parties do not have altruistic behavior, the final stable values of both parties' profits are higher when both parties have altruistic behavior, and when the retailer shares the emission reduction costs for the manufacturer, the final stable values will be further improved.

Combining Figures 5 and 6, it can be found that when consumers' low-carbon consumption behavior is affected by the reference low-carbon effect, it will damage the profits of both manufacturers and retailers, forcing manufacturers to reduce emissions reduction investment and retailers to reduce low-carbon publicity investment, which will directly do harm to low-carbon level and low-carbon goodwill and eventually adversely affect the low-carbon product market. However, when consumers' low-carbon consumption behavior is affected by the reference low-carbon effect, the altruistic behavior of both parties can weaken the adverse effect of the reference low-carbon effect to a certain extent, and the weakening ability is proportional to the altruistic intensity of both parties. In addition, if the retailer shares the emission reduction costs for the manufacturer on this basis, this weakening effect can be further strengthened, and the damaging effect on the profits of both parties due to consumers' reference low-carbon effect can be minimized.

\section{Conclusions and Discussion}

In the context of the low-carbon economy, when consumers buy low-carbon products, they are always affected by the reference low-carbon effect. At the same time, supply chain members are not completely rational when making emission reduction decisions, and they are always affected by altruistic behavior. Based on the above background, this paper introduces consumers' reference low-carbon effect and the supply chain members' altruistic behavior into the joint emission reduction issue of a supply chain, and respectively constructs decision-making models under four decision scenarios: centralized scenario, decentralized scenario, decentralized scenario with altruistic behavior and decentralized scenario with altruistic behavior and a cost-sharing contract. Then this paper uses differential game theory and Bellman continuous dynamic programming theory to solve the equilibrium solutions under the four decision scenarios in turn, and discusses the impact of the supply chain members' altruistic behavior, consumers' reference low-carbon effect, cost-sharing contract and related key parameters on supply chain members' equilibrium strategies and performance from a long-term perspective. After mathematical derivation and numerical analysis, the following conclusions can be obtained:

Compared with both parties not having altruistic behavior, manufacturers' emission reduction 
investment, retailers' low-carbon publicity investment, steady-state low-carbon level, steady-state low-carbon goodwill and both parties' profits will increase when both parties have altruistic behavior. Both parties' profits will increase with the increase in the altruistic intensity of the other party, and will decrease with the increase in the altruistic intensity of itself, while both parties' utilities will increase with the increase in the altruistic intensity of either party. In particular, when both parties are completely altruistic, both the emission reduction investment of the manufacturer and the low-carbon publicity investment of the retailer can reach the level under centralized decision scenario, and the sum of the two parties' profits can also reach the overall profit of the supply chain under centralized decision scenario. Therefore, the supply chain is fully coordinated at this time.

Under decentralized scenario with altruistic behavior and a cost-sharing contract, retailers sharing emission reduction costs for manufacturers will help promote manufacturers to increase emission reduction investment, and as the cost-sharing ratio increases, manufacturers' emission reduction investment will continue to increase, but will not affect their own low-carbon publicity investment. The optimal emission reduction cost-sharing ratio provided by the retailer to the manufacturer is only related to the marginal revenue and altruistic intensity of both parties, and will decrease as the manufacturer's marginal revenue and altruistic intensity increase, but will increase as the retailer's marginal revenue and altruistic intensity increase. In particular, when the retailer is completely altruistic, both the manufacturer's emission reduction investment and the retailer's low-carbon publicity investment can reach the level under centralized scenario, the sum of the two parties' profits can also reach the overall profit of the supply chain under centralized scenario, therefore, the emission reduction cost-sharing contract realizes the perfect coordination of the supply chain at this time.

When both parties have altruistic behavior and the retailer provides the manufacturer with a emission reduction cost-sharing contract, both parties' profits and utilities can be further improved compared with no cost-sharing contract, and the profit increment and utility increment will continue to decrease as the manufacturer's altruistic intensity continues to increase, but will increase as the retailer's altruistic intensity continues to increase. In particular, when the retailer is completely altruistic and the manufacturer is close to self-interest, both parties can obtain the greatest profit and utility increment.

Consumers' reference low-carbon effect will damage the profits of both manufacturers and retailers, force manufacturers to reduce emission reduction investment and retailers to reduce low-carbon publicity investment, which will directly do harm to low-carbon level and low-carbon goodwill and eventually adversely affect the low-carbon product market.

The altruistic behavior of manufacturers and retailers can not only improve both parties' profits, utilities and the overall profit of supply chain, but also weaken the harmful impact of consumers' reference low-carbon effect on both parties' profits and the overall profit of the supply chain. Furthermore, it can also encourage both parties to increase emission reduction investment, and produce a continuous and stable positive social effect. In short, from a long-term perspective, it is a positive social behavior.

Based on the above conclusions, in order to provide more references for supply chain companies to make suitable emission reduction decisions, we give the following management insights:

Both the government and supply chain enterprises should strengthen the propaganda of the low-carbon economy through a variety of ways, and advocate a low-carbon culture, so that it can raise the public's awareness of environmental protection and promote low-carbon consumption. In addition, the government should establish a scientific low-carbon product certification system and increase the supervision of low-carbon certified products, so that it can strengthen consumers' belief in low-carbon 
products, avoid their blindly relying on low-carbon goodwill when purchasing low-carbon products, reduce the negative impact of consumers' reference low-carbon effect on the emission reduction enthusiasm of supply chain members , and maintain a good low-carbon product market to a certain extent.

In the context of the low-carbon economy, in order to maintain continuous market competitiveness, supply chain enterprises should form altruistic concepts when conducting joint emission reductions, and actively practice altruistic behavior in a variety of ways. In addition, they should also focus on making emission reduction decisions from a long-term perspective at the same time. Because altruistic behavior can not only encourage enterprises to increase their investment in emission reduction and low-carbon publicity, but can also effectively improve profits and utilities of enterprises, and produce a continuous and positive social effect and a steady stream of social welfare from a long-term perspective.

Manufacturers and retailers in the supply chain should also actively cooperate by sharing emission reduction costs with each other, because a cost-sharing contract can not only increase the profits of both parties, but also increase the utilities of both parties when supply chain members have altruistic behavior. At the same time, since the cost-sharing ratio is proportional to supply chain members' own marginal profits and altruistic intensity, in order to further enhance the cooperation level between the two parties, manufacturers and retailers should pay more attention to their own marginal profits and altruistic intensity to ensure that more profit and utility increments can be obtained under the cost-sharing contract.

Even though this paper has made some contributions to the dynamic decision-making of joint emission reduction in the supply chain, this paper still has some shortcomings that need to be explored in future studies. This paper only considers supply chain members' altruistic behavior, but in real life, supply chain members may be affected by multiple behaviors at the same time when they make emission reduction decisions due to the impact of multiple factors. Therefore, future studies can explore the influence of other behavioral factors on the joint emission reduction decisions of supply chain members from a long-term perspective. In addition, this paper does not consider the influence of price factors when constructing the demand function model, and assumes that the marginal profits of manufacturers and retailers are constant. Therefore, how do consumers' reference low-carbon effect and supply chain members' altruistic behavior affect their decisions and performance when the price factors are considered? Some related issues need further discussion.

\section{Acknowledgments}

All authors thank the reviewers and editors for their work.

\section{Authors Contributions}

Zhang ZY. proposed the topic, designed the research content, and revised the final version of the paper. Yu LY was involved in the methodology, simulation analysis and provided guidance during the writing of the paper. All authors have read and agreed to the published version of the manuscript.

\section{Funding}

This study was funded by National Natural Science Foundation of China (grant number 12071280; 11671250) and Shanghai Planning Project of Philosophy and Social Sciences of China (grant number 2020BGL023).

\section{Data availability}

All data generated or analyzed during this study are included in this paper.

\section{Declarations}


Ethics approval and consent to participate: Not applicable.

Consent for publication: Not applicable.

Competing interests: The authors declare no competing interests.

\section{Appendix}

Proof of Theorem 3. Based on the optimal control theory and equation (12), the retailer's objective function at time $\mathrm{t}$ is:

$$
\begin{aligned}
& J_{r}^{a}(T, G, t)=\max _{A} \int_{t}^{\infty} e^{-\rho s}\left[\left(\tau_{r} \pi_{1}+\pi_{2}\right)[v+\alpha T+k A+(\mu-\alpha \varepsilon) G]-\frac{\eta_{r} A^{2}}{2}-\frac{\eta_{m} \tau_{r} E^{2}}{2}\right] d s \\
& =e^{-\rho t} \max _{A} \int_{t}^{\infty} e^{-\rho(s-t)}\left[\left(\tau_{r} \pi_{1}+\pi_{2}\right)[v+\alpha T+k A+(\mu-\alpha \varepsilon) G]-\frac{\eta_{r} A^{2}}{2}-\frac{\eta_{m} \tau_{r} E^{2}}{2}\right] d s
\end{aligned}
$$

$$
\text { let } V_{r}(T, G)=\max _{A} \int_{t}^{\infty} e^{-\rho(s-t)}\left[\left(\tau_{r} \pi_{1}+\pi_{2}\right)[v+\alpha T+k A+(\mu-\alpha \varepsilon) G]-\frac{\eta_{r} A^{2}}{2}-\frac{\eta_{m} \tau_{r} E^{2}}{2}\right] d s \text {, we }
$$
can ger the retailer's optimal profit function at time t is: $J_{r}^{a}(T, G, t)=e^{-\rho t} V_{r}(T, G)$.

Here, we can get the available HJB equation:

$$
\rho V_{r}(T, G)+\rho \tau_{r} V_{m}(T, G)=\max _{A}\left[\begin{array}{c}
\left(\tau_{r} \pi_{1}+\pi_{2}\right)[v+\alpha T+k A+(\mu-\alpha \varepsilon) G]+\left(\frac{\partial V_{r}}{\partial T}+\tau_{r} \frac{\partial V_{m}}{\partial T}\right)(\gamma E-\delta T) \\
+\left(\frac{\partial V_{r}}{\partial G}+\tau_{r} \frac{\partial V_{m}}{\partial G}\right)(\phi T+\lambda A-\vartheta G)-\frac{\eta_{r} A^{2}}{2}-\frac{\eta_{m} \tau_{r} E^{2}}{2}
\end{array}\right]
$$

By solving the first-order partial derivative of $A$, we can get:

$$
A=\frac{\left(\tau_{r} \pi_{1}+\pi_{2}\right) k+\lambda\left(\frac{\partial V_{r}}{\partial G}+\tau_{r} \frac{\partial V_{m}}{\partial G}\right)}{\eta_{r}}
$$

According to Equation (11), the optimal profit function of the manufacturer at time $\mathrm{t}$ can be expressed as: $J_{m}^{a}(T, G, t)=e^{-\rho t} V_{m}(T, G)$.

Let $V_{m}(T, G)=\max _{E} \int_{t}^{\infty} e^{-\rho(s-t)}\left[\left(\tau_{m} \pi_{2}+\pi_{1}\right)[v+\alpha T+k A+(\mu-\alpha \varepsilon) G]-\frac{\eta_{r} \tau_{m} A^{2}}{2}-\frac{\eta_{m} E^{2}}{2}\right] d s$.

Then, we can get the available HJB equation:

$$
\rho V_{m}(T, G)+\rho \tau_{m} V_{r}(T, G)=\max _{E}\left[\begin{array}{l}
\left(\tau_{m} \pi_{2}+\pi_{1}\right)[\nu+\alpha T+k A+(\mu-\alpha \varepsilon) G]-\frac{\eta_{r} \tau_{m} A^{2}}{2}-\frac{\eta_{m} E^{2}}{2} \\
+\left(\tau_{m} \frac{\partial V_{r}}{\partial T}+\frac{\partial V_{m}}{\partial T}\right)(\gamma E-\delta T)+\left(\tau_{m} \frac{\partial V_{r}}{\partial G}+\frac{\partial V_{m}}{\partial G}\right)(\phi T+\lambda A-\vartheta G)
\end{array}\right]
$$

By solving the first-order partial derivative of $E$, we can get:

$$
E=\frac{\gamma\left(\tau_{m} \frac{\partial V_{r}}{\partial T}+\frac{\partial V_{m}}{\partial T}\right)}{\eta_{m}}
$$

Sum up through sorting, we can obtain: 


$$
\begin{aligned}
& \rho V_{r}+\rho \tau_{r} V_{m}=\left[\left(\tau_{r} \pi_{1}+\pi_{2}\right)(\mu-\alpha \varepsilon)-\vartheta\left(\frac{\partial V_{r}}{\partial G}+\tau_{r} \frac{\partial V_{m}}{\partial G}\right)\right] G+\left[\begin{array}{l}
\left(\tau_{r} \pi_{1}+\pi_{2}\right) \alpha+\phi\left(\frac{\partial V_{r}}{\partial G}+\tau_{r} \frac{\partial V_{m}}{\partial G}\right) \\
-\delta\left(\frac{\partial V_{r}}{\partial T}+\tau_{r} \frac{\partial V_{m}}{\partial T}\right)
\end{array}\right] T \\
& +\frac{\left[\left(\tau_{r} \pi_{1}+\pi_{2}\right) k+\lambda\left(\frac{\partial V_{r}}{\partial G}+\tau_{r} \frac{\partial V_{m}}{\partial G}\right)\right]^{2}}{2 \eta_{r}}+\frac{\gamma^{2}\left(\tau_{m} \frac{\partial V_{r}}{\partial T}+\frac{\partial V_{m}}{\partial T}\right)\left[2 \frac{\partial V_{r}}{\partial T}+\tau_{r} \frac{\partial V_{m}}{\partial T}-\tau_{r} \tau_{m} \frac{\partial V_{r}}{\partial T}\right]}{2 \eta_{m}}+\left(\tau_{r} \pi_{1}+\pi_{2}\right) v
\end{aligned}
$$

$$
\begin{aligned}
& \rho V_{m}+\rho \tau_{m} V_{r}=\left[\left(\tau_{m} \pi_{2}+\pi_{1}\right)(\mu-\alpha \varepsilon)-\vartheta\left(\tau_{m} \frac{\partial V_{r}}{\partial G}+\frac{\partial V_{m}}{\partial G}\right)\right] G+\left[\begin{array}{l}
\alpha\left(\tau_{m} \pi_{2}+\pi_{1}\right)+\phi\left(\tau_{m} \frac{\partial V_{r}}{\partial G}+\frac{\partial V_{m}}{\partial G}\right) \\
-\delta\left(\tau_{m} \frac{\partial V_{r}}{\partial T}+\frac{\partial V_{m}}{\partial T}\right)
\end{array}\right] T+\left(\tau_{m} \pi_{2}+\pi_{1}\right) \nu \\
& +\frac{\left[\left(\tau_{r} \pi_{1}+\pi_{2}\right) k+\lambda\left(\frac{\partial V_{r}}{\partial G}+\tau_{r} \frac{\partial V_{m}}{\partial G}\right)\right]\left[\begin{array}{l}
2\left(\tau_{m} \pi_{2}+\pi_{1}\right) k+2 \lambda\left(\tau_{m} \frac{\partial V_{r}}{\partial G}+\frac{\partial V_{m}}{\partial G}\right) \\
\left.-\tau_{m}\left(\tau_{r} \pi_{1}+\pi_{2}\right) k-\tau_{m} \lambda\left(\frac{\partial V_{r}}{\partial G}+\tau_{r} \frac{\partial V_{m}}{\partial G}\right)\right]
\end{array}\right]+\frac{\gamma^{2}\left(\tau_{m} \frac{\partial V_{r}}{\partial T}+\frac{\partial V_{m}}{\partial T}\right)^{2}}{2 \eta_{m}}}{2 \eta_{r}}
\end{aligned}
$$

830 We assume that $V_{r}(T, G)$ and $V_{m}(T, G)$ satisfy the following expression, respectively:

$$
\begin{aligned}
& V_{r}(T, G)=a_{4} T+b_{4} G+c_{4} \\
& V_{m}(T, G)=a_{5} T+b_{5} G+c_{5}
\end{aligned}
$$

831 Then, we can get:

$$
\begin{aligned}
& \int \rho a_{4}+\rho \tau_{r} a_{5}=\left(\tau_{r} \pi_{1}+\pi_{2}\right) \alpha+\phi\left(b_{4}+\tau_{r} b_{5}\right)-\delta\left(a_{4}+\tau_{r} a_{5}\right) \\
& \rho b_{4}+\rho \tau_{r} b_{5}=\left(\tau_{r} \pi_{1}+\pi_{2}\right)(\mu-\alpha \varepsilon)-\vartheta\left(b_{4}+\tau_{r} b_{5}\right) \\
& \rho c_{4}+\rho \tau_{r} c_{5}=\left(\tau_{r} \pi_{1}+\pi_{2}\right) v+\frac{\left[\left(\tau_{r} \pi_{1}+\pi_{2}\right) k+\lambda\left(b_{4}+\tau_{r} b_{5}\right)\right]^{2}}{2 \eta_{r}}+\frac{\gamma^{2}\left(\tau_{m} a_{4}+a_{5}\right)\left(2 a_{4}+\tau_{r} a_{5}-\tau_{r} \tau_{m} a_{4}\right)}{2 \eta_{m}} \\
& \left\{\rho a_{5}+\rho \tau_{m} a_{4}=\alpha\left(\tau_{m} \pi_{2}+\pi_{1}\right)+\phi\left(\tau_{m} b_{4}+b_{5}\right)-\delta\left(\tau_{m} a_{4}+a_{5}\right)\right. \\
& \rho b_{5}+\rho \tau_{m} b_{4}=\left(\tau_{m} \pi_{2}+\pi_{1}\right)(\mu-\alpha \varepsilon)-\vartheta\left(\tau_{m} b_{4}+b_{5}\right) \\
& \rho c_{5}+\rho \tau_{m} c_{4}=\left(\tau_{m} \pi_{2}+\pi_{1}\right) v+\frac{\left[\left(\tau_{r} \pi_{1}+\pi_{2}\right) k+\lambda\left(b_{4}+\tau_{r} b_{5}\right)\right]\left(\begin{array}{l}
2\left(\tau_{m} \pi_{2}+\pi_{1}\right) k+2 \lambda\left(\tau_{m} b_{4}+b_{5}\right) \\
-\tau_{m}\left(\tau_{r} \pi_{1}+\pi_{2}\right) k-\tau_{m} \lambda\left(b_{4}+\tau_{r} b_{5}\right)
\end{array}\right)}{2 \eta_{r}}+\frac{\gamma^{2}\left(a_{5}+\tau_{m} a_{4}\right)^{2}}{2 \eta_{m}}
\end{aligned}
$$

832 By solving the equations, we can obtain:

$$
a_{4}^{*}=\frac{\pi_{2}[\phi(\mu-\alpha \varepsilon)+\alpha(\rho+\vartheta)]}{(\rho+\vartheta)(\rho+\delta)}, a_{5}^{*}=\frac{\pi_{1}[\phi(\mu-\alpha \varepsilon)+\alpha(\rho+\vartheta)]}{(\rho+\vartheta)(\rho+\delta)}, b_{4}^{*}=\frac{\pi_{2}(\mu-\alpha \varepsilon)}{\rho+\vartheta}, b_{5}^{*}=\frac{\pi_{1}(\mu-\alpha \varepsilon)}{\rho+\vartheta},
$$




$$
\begin{aligned}
& c_{4}^{*}=\frac{1}{\rho}\left\{\pi_{2} v+\frac{\gamma^{2}\left(\tau_{m} \pi_{2}+\pi_{1}\right) \pi_{2}[\phi(\mu-\alpha \varepsilon)+\alpha(\rho+\vartheta)]^{2}}{\eta_{m}(\rho+\vartheta)^{2}(\rho+\delta)^{2}}+\frac{\left[\pi_{2}^{2}-\left(\tau_{r} \pi_{1}\right)^{2}\right][(\rho+\vartheta) k+\lambda(\mu-\alpha \varepsilon)]^{2}}{2 \eta_{r}(\rho+\vartheta)^{2}}\right\}, \\
& c_{5}^{*}=\frac{1}{\rho}\left\{\pi_{1} v+\frac{\gamma^{2}\left[\pi_{1}^{2}-\left(\tau_{m} \pi_{2}\right)^{2}\right][\phi(\mu-\alpha \varepsilon)+\alpha(\rho+\vartheta)]^{2}}{2 \eta_{m}(\rho+\vartheta)^{2}(\rho+\delta)^{2}}+\frac{\left(\tau_{r} \pi_{1}+\pi_{2}\right) \pi_{1}[(\rho+\vartheta) k+\lambda(\mu-\alpha \varepsilon)]^{2}}{\eta_{r}(\rho+\vartheta)^{2}}\right\} .
\end{aligned}
$$

Finally, the both parties' optimal decisions can be obtained:

$$
\left\{\begin{array}{l}
E^{a^{*}}=\frac{\gamma\left(\tau_{m} \pi_{2}+\pi_{1}\right)[\phi(\mu-\alpha \varepsilon)+\alpha(\rho+\vartheta)]}{\eta_{m}(\rho+\vartheta)(\rho+\delta)} \\
A^{a^{*}}=\frac{\left(\tau_{r} \pi_{1}+\pi_{2}\right)[k(\rho+\vartheta)+\lambda(\mu-\alpha \varepsilon)]}{\eta_{r}(\rho+\vartheta)}
\end{array}\right.
$$

Theorem 3 is proved.

\section{References}

Bassi M, Pagnozzi M, Piccolo S. Optimal contracting with altruism and reciprocity [J]. Research in Economics, 2014, 68(1): 27-38.

Bendoly E, Donohue K, Schultz K L. Behavior in operations management: Assessing recent findings and revisiting old assumptions [J]. Journal of Operations Management, 2006, 24(6): 737-752.

Benjaafar S, Li Y Z, Daskin M. Carbon Footprint and the Management of Supply Chains: Insights From Simple Models [J]. Ieee Transactions on Automation Science and Engineering, 2013, 10(1): 99-116.

Bertinelli L, Camacho C, Zou B T. Carbon capture and storage and transboundary pollution: A differential game approach [J]. European Journal of Operational Research, 2014, 237(2): 721-728.

Cao K Y, Xu B, He Y, et al. Optimal carbon reduction level and ordering quantity under financial constraints [J]. International Transactions in Operational Research, 2020, 27(5): 2270-2293.

Cheng X, Wang C X, Xu L. Decisions of Pricing and Reduction Rate in Supply Chain Considering Altruism Preference [J]. Industrial Engineering and Management, 2018, 23(02): 159-166.

Dye C Y, Yang C T, Wu C C. Joint dynamic pricing and preservation technology investment for an integrated supply chain with reference price effects [J]. Journal of the Operational Research Society, 2018, 69(6): 811-824.

Fan R G, Lin J C, Zhu K W. Study of game models and the complex dynamics of a low-carbon supply chain with an altruistic retailer under consumers' low-carbon preference [J]. Physica a-Statistical Mechanics and Its Applications, 2019, 528, 121460.

Ghosh D, Shah J. Supply chain analysis under green sensitive consumer demand and cost sharing contract [J]. International Journal of Production Economics, 2015, 164,319-329.

Han Q, Wang Y Y. Decision and Coordination in a Low-Carbon E-Supply Chain Considering the Manufacturer's Carbon Emission Reduction Behavior [J]. Sustainability, 2018, 10(5): 1686.

He Y Y, Zhang J, Gou Q L, et al. Supply chain decisions with reference quality effect under the O2O environment [J]. Annals of Operations Research, 2018, 268(1-2): 273-292.

He Y, Zhang J, Gou Q, et al. Supply chain decisions with reference quality effect under the $\mathrm{O} 2 \mathrm{O}$ environment [J]. Annals of Operations Research, 2017, 1: 1-20. 
Hellofs L L, Jacobson R. Market share and customers' perceptions of quality: When can firms grow their way to higher versus lower quality? [J]. Journal of Marketing, 1999, 63(1): 16-25.

Huang H, Zhang J, Ren X, et al. Greenness and Pricing Decisions of Cooperative Supply Chains Considering Altruistic Preferences [J]. International Journal of Environmental Research and Public Health, 2019, 16(1): 51.

Kopalle P K, Winer R S. A dynamic model of reference price and expected quality [J]. Marketing Letters, 1996, 7(1): 41-52.

Li Q Q, Xiao T J, Qiu Y Z. Price and carbon emission reduction decisions and revenue-sharing contract considering fairness concerns [J]. Journal of Cleaner Production, 2018, 190,303-314.

Lin Z B. Price promotion with reference price effects in supply chain [J]. Transportation Research Part E-Logistics and Transportation Review, 2016, 85,52-68.

Liu G W, Sethi S P, Zhang J X. Myopic vs. far-sighted behaviours in a revenue-sharing supply chain with reference quality effects [J]. International Journal of Production Research, 2016, 54(5): 1334-1357.

Liu M L, Li Z H, Anwar S, et al. Supply chain carbon emission reductions and coordination when consumers have a strong preference for low-carbon products $[\mathrm{J}]$. Environmental Science and Pollution Research, 2021.

Loch C H, Wu Y Z. Social Preferences and Supply Chain Performance: An Experimental Study [J]. Management Science, 2008, 54(11): 1835-1849.

Lou G X, Zhang Y M, Wan N. Dynamic pricing and emission abatement with reference effect. [J]. Control and decision, 2018, 33(09), 1667-1676.

Popescu I, Wu Y Z. Dynamic pricing strategies with reference effects [J]. Operations Research, 2007, 55(3): 413-429.

Pu X J, Song Z P, Han G H. Competition among Supply Chains and Governmental Policy: Considering Consumers' Low-Carbon Preference [J]. International Journal of Environmental Research and Public Health, 2018, 15(9): 1985.

Wang D D, Liu Y. Supply Chain Pricing Decision Considering Altruistic Preferences and Carbon Emission Reduction Efforts[J]. Industrial Engineering and Management, 2020,25(6),1-15.

Wang Q P, Zhao D Z, He L F. Contracting emission reduction for supply chains considering market low-carbon preference [J]. Journal of Cleaner Production, 2016, 120,72-84.

Wang Z R, Brownlee A E I, Wu Q H. Production and joint emission reduction decisions based on two-way cost-sharing contract under cap-and-trade regulation [J]. Computers \& Industrial Engineering, 2020.

Xia L J, Bai Y W, Ghose S, et al. Differential game analysis of carbon emissions reduction and promotion in a sustainable supply chain considering social preferences $[\mathrm{J}]$. Annals of Operations Research, 2020.

Xia L J, Guo T T, Qin J J, et al. Carbon emission reduction and pricing policies of a supply chain considering reciprocal preferences in cap-and-trade system [J]. Annals of Operations Research, 2018, 268(1-2): 149-175.

Ye T, Guan Z M, Tao J, et al. Dynamic Optimization and Coordination About Joint Emission Reduction in a Supply Chain Considering Consumer Preference to Low Carbon and Reference Low-carbon Level Effect. [J]. Chinese Journal of Management Science, 2017, 25(10), 52-61.

Yu S, Hou Q. Supply Chain Investment in Carbon Emission-Reducing Technology Based on Stochasticity and Low-Carbon Preferences [J]. Complexity, 2021. 
Zhang J, Chiang W Y K, Liang L. Strategic pricing with reference effects in a competitive supply chain [J]. Omega-International Journal of Management Science, 2014, 44,126-135.

Zhang J, Gou Q L, Liang L, et al. Supply chain coordination through cooperative advertising with reference price effect [J]. Omega-International Journal of Management Science, 2013, 41(2): 345-353.

Zhang L H, Wang J G, You J X. Consumer environmental awareness and channel coordination with two substitutable products [J]. European Journal of Operational Research, 2015, 241(1): 63-73.

Zhang L H, Xue B W, Liu X Y. Carbon Emission Reduction with Regard to Retailer's Fairness Concern and Subsidies [J]. Sustainability, 2018, 10(4): 1209.

Zhang X L, Xiu G Y, Shahzad F, et al. The Impact of Equity Financing on the Performance of Capital-Constrained Supply Chain under Consumers' Low-Carbon Preference [J]. International Journal of Environmental Research and Public Health, 2021, 18(5): 2329.

Zhang Y, Li J Y, Xu B. Designing Buy-Online-and-Pick-Up-in-Store (BOPS) Contract of Dual-Channel Low-Carbon Supply Chain considering Consumers' Low-Carbon Preference [J]. Mathematical Problems in Engineering, 2020.

Zhang Z C, Zhang Q, Liu Z, et al. Static and Dynamic Pricing Strategies in a Closed-Loop Supply Chain with Reference Quality Effects [J]. Sustainability, 2018, 10(1): 157.

Zhang Z Y, Fu D X, Zhou Q. Optimal Decisions of a Green Supply Chain under the Joint Action of Fairness Preference and Subsidy to the Manufacturer [J]. Discrete Dynamics in Nature and Society, 2020 .

Zhang Z Y, Yu L Y. Dynamic Optimization and Coordination of Cooperative Emission Reduction in a Dual-Channel Supply Chain Considering Reference Low-Carbon Effect and Low-Carbon Goodwill [J]. International Journal of Environmental Research and Public Health, 2021, 18(2): 539.

Zhou Y J, Bao M J, Chen X H, et al. Co-op advertising and emission reduction cost sharing contracts and coordination in low-carbon supply chain based on fairness concerns [J]. Journal of Cleaner Production, 2016, 133,402-413. 
Figures

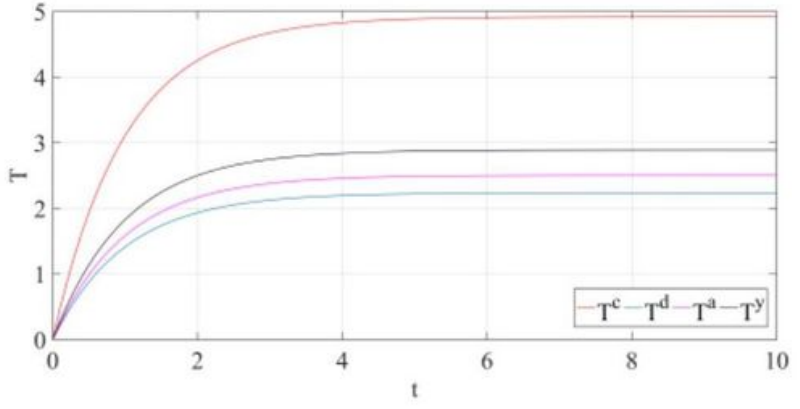

(a)

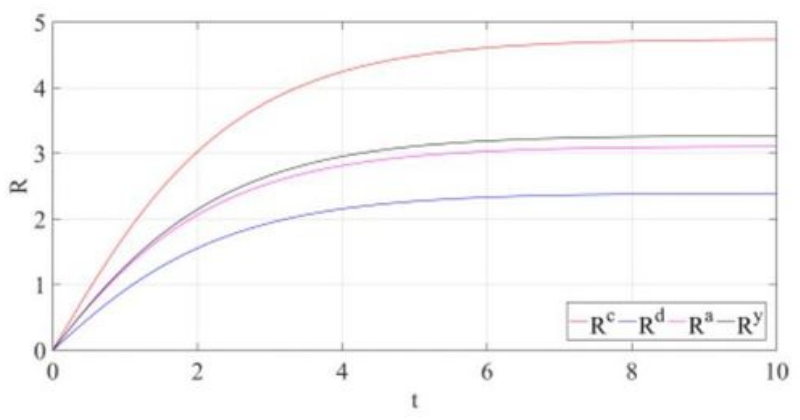

(c)

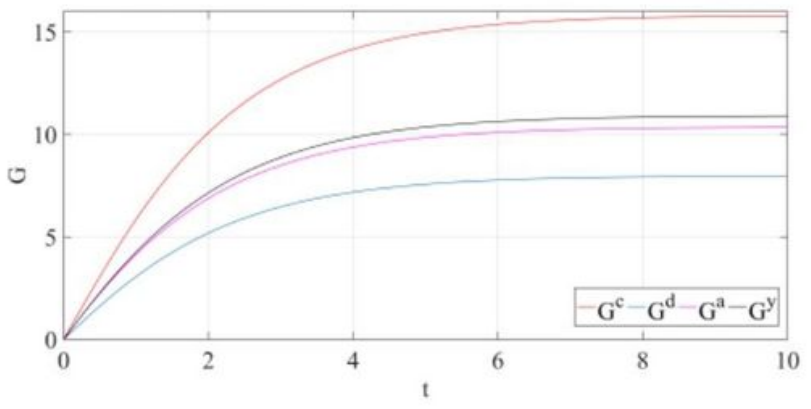

(b)

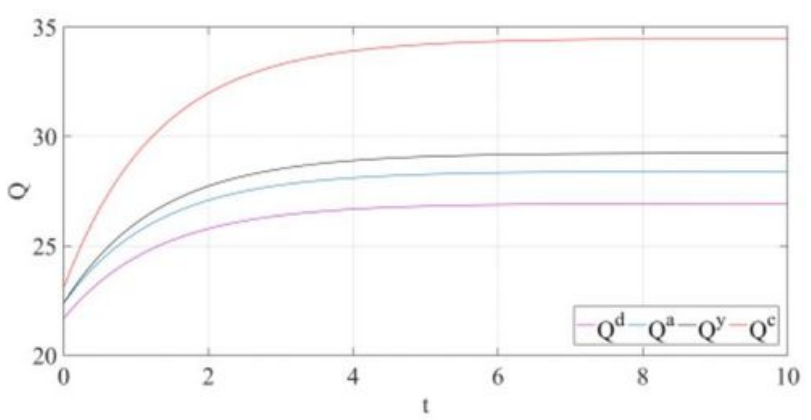

(d)

\section{Figure 1}

Please see the Manuscript PDF file for the complete figure caption 


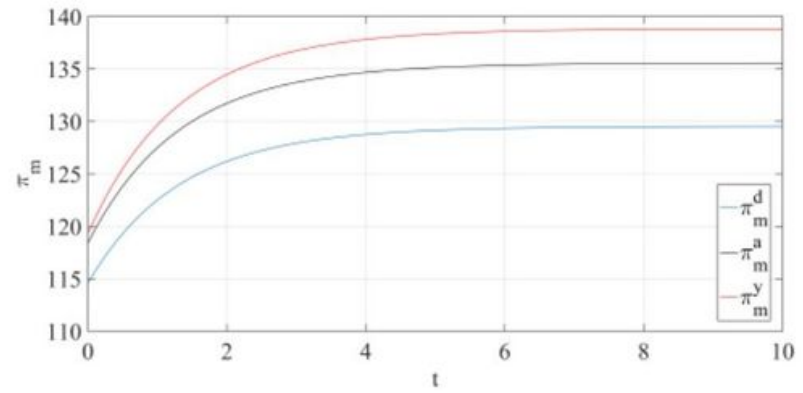

(a)

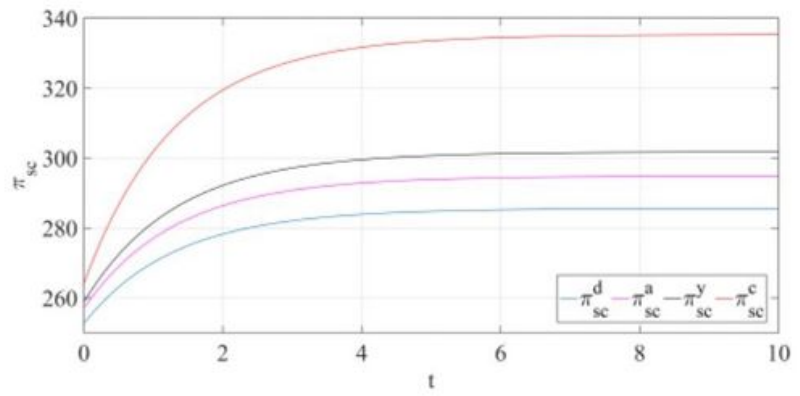

(c)

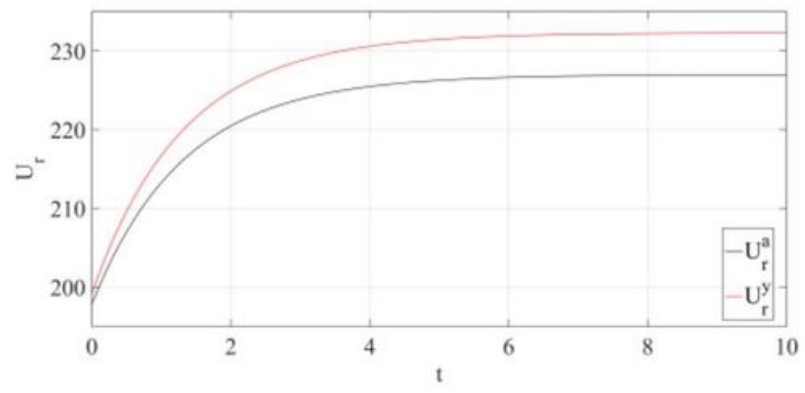

(e)

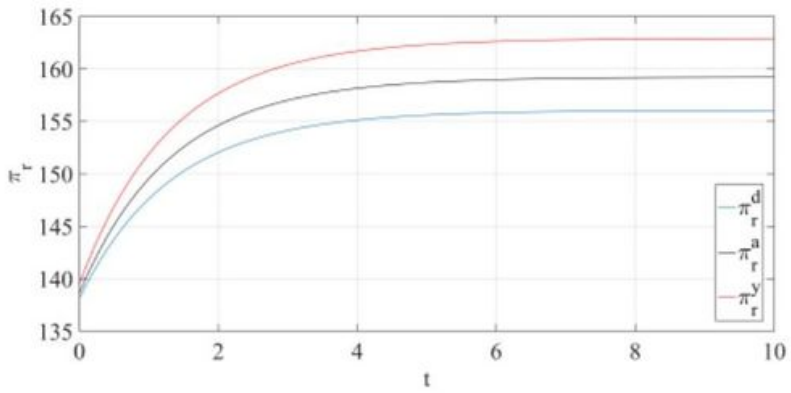

(b)

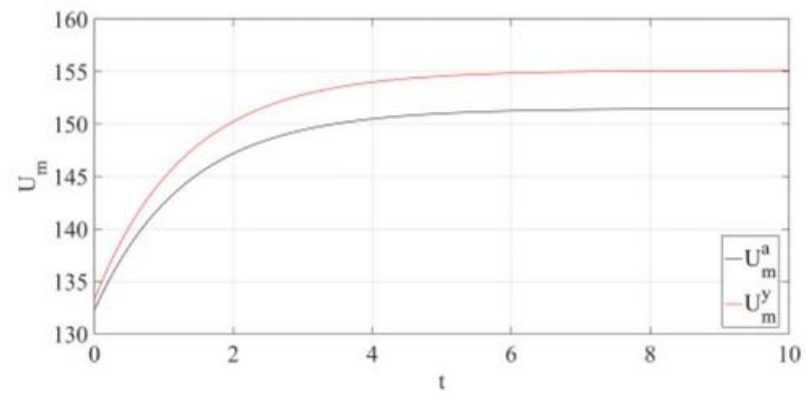

(d)

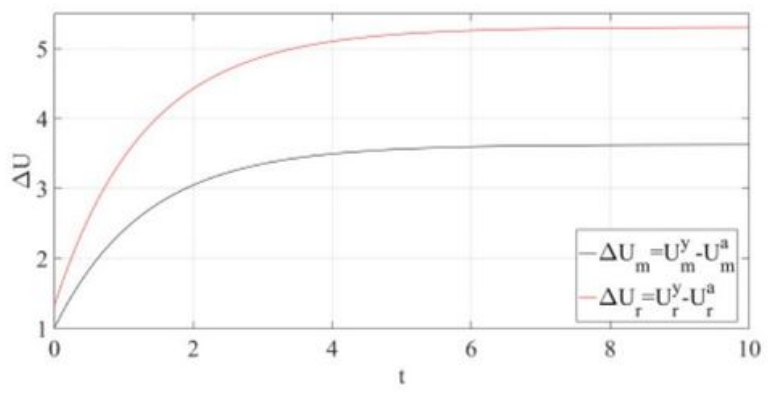

(f)

Figure 2

Please see the Manuscript PDF file for the complete figure caption 


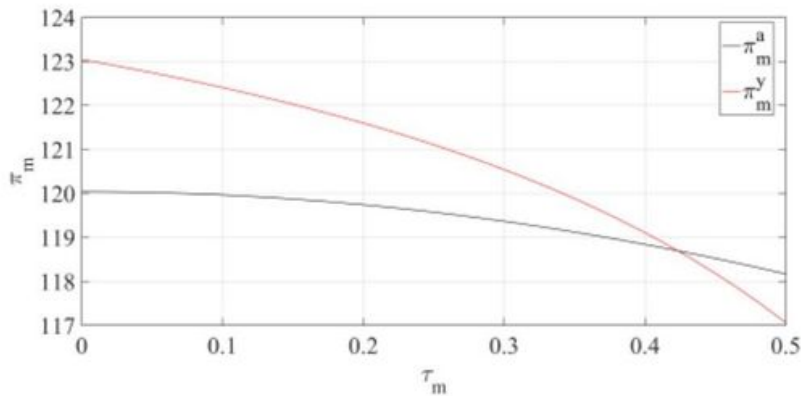

(a)

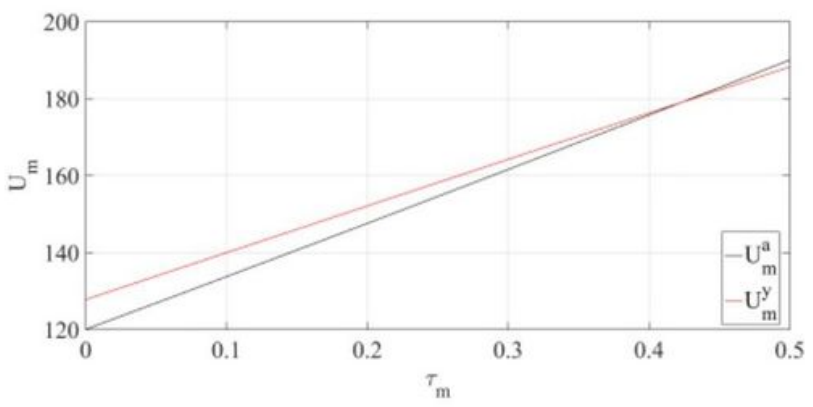

(c)

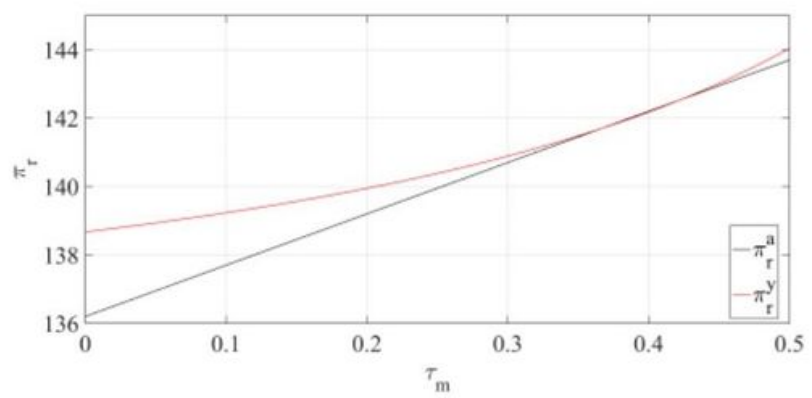

(b)

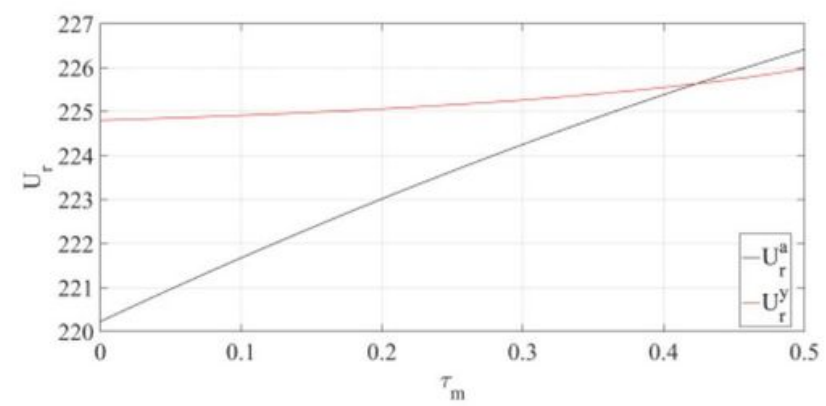

(d)

\section{Figure 3}

Please see the Manuscript PDF file for the complete figure caption

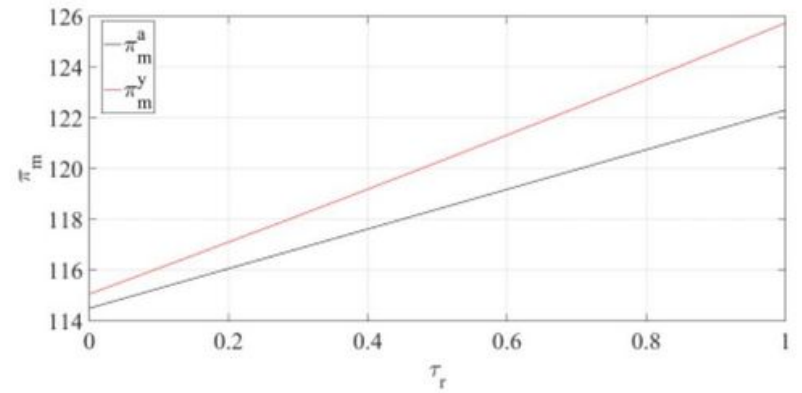

(a)

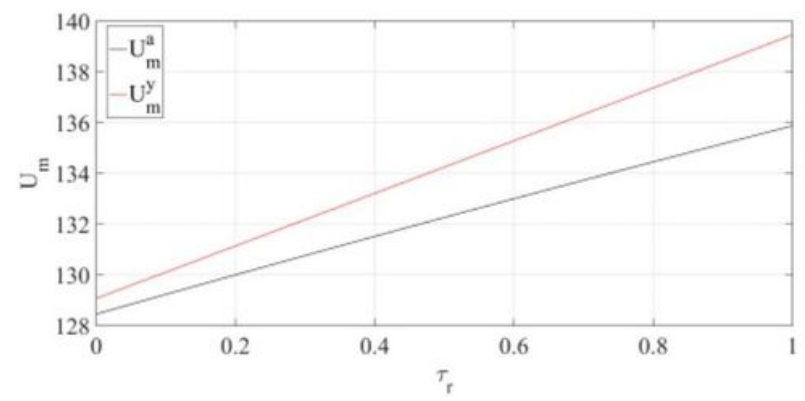

(c)

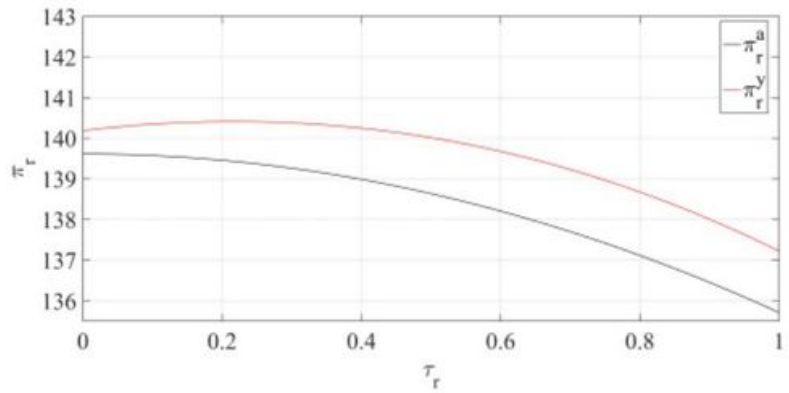

(b)

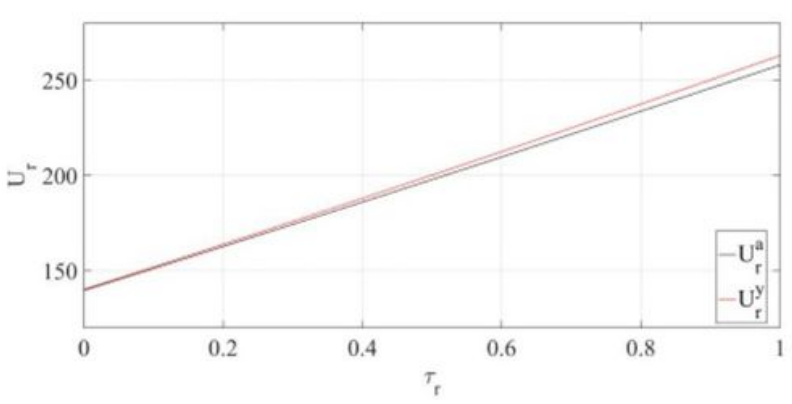

(d)

\section{Figure 4}

Please see the Manuscript PDF file for the complete figure caption 


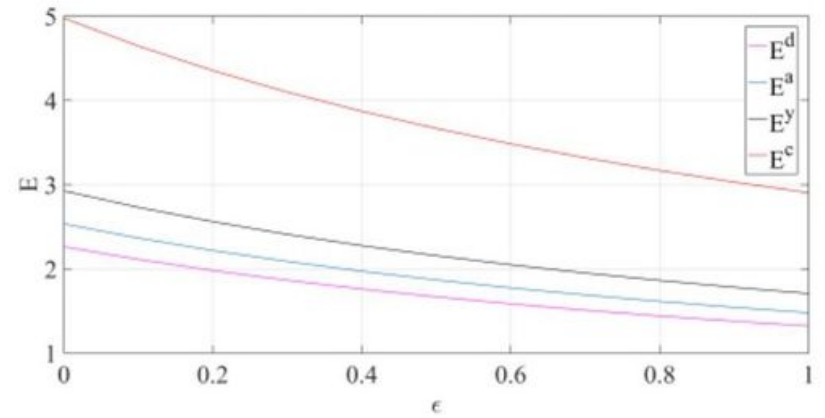

(a)

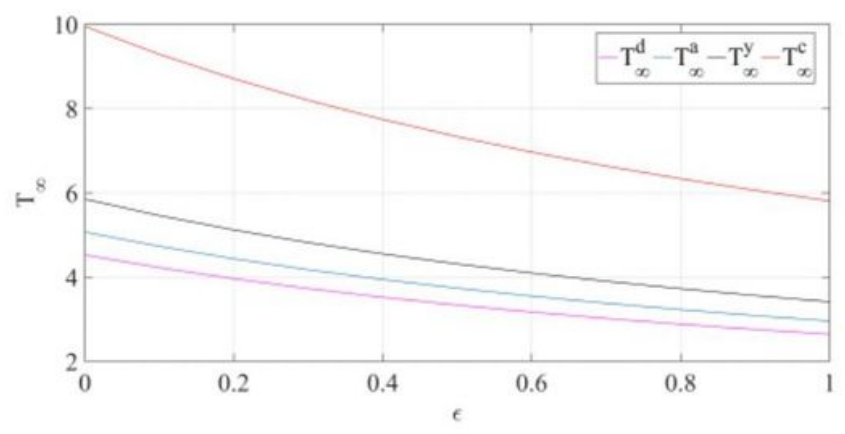

(c)

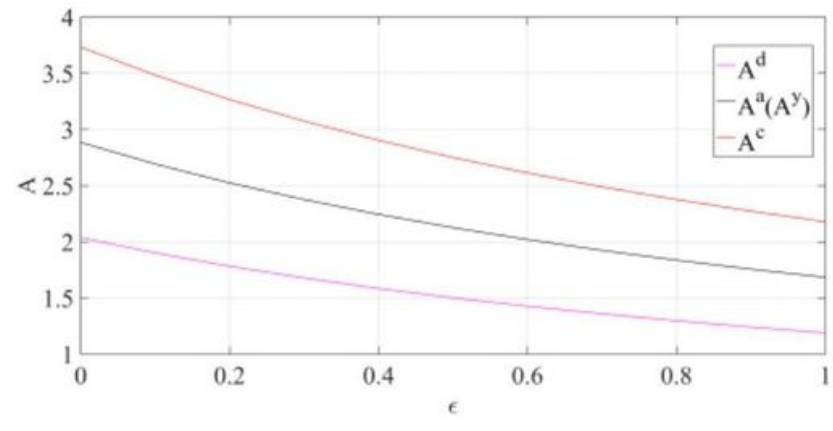

(b)

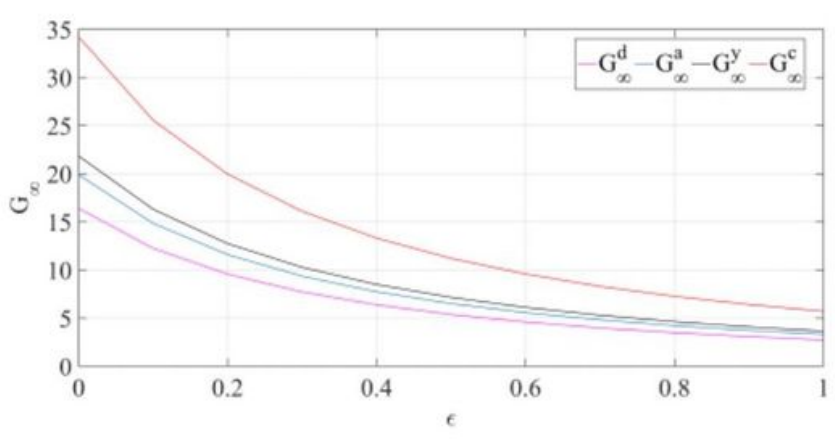

(d)

\section{Figure 5}

Please see the Manuscript PDF file for the complete figure caption

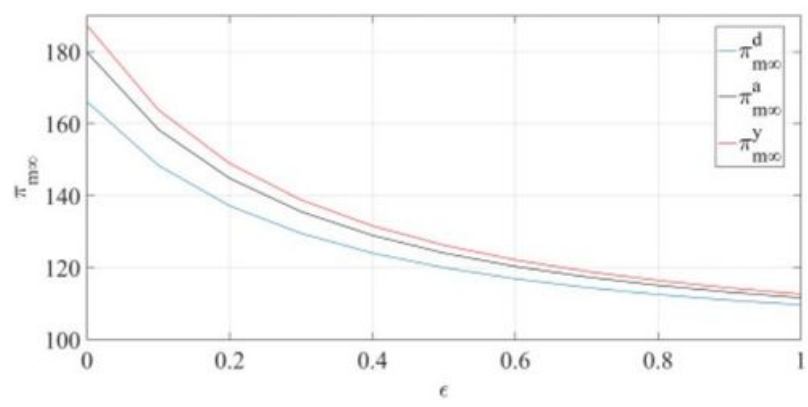

(a)

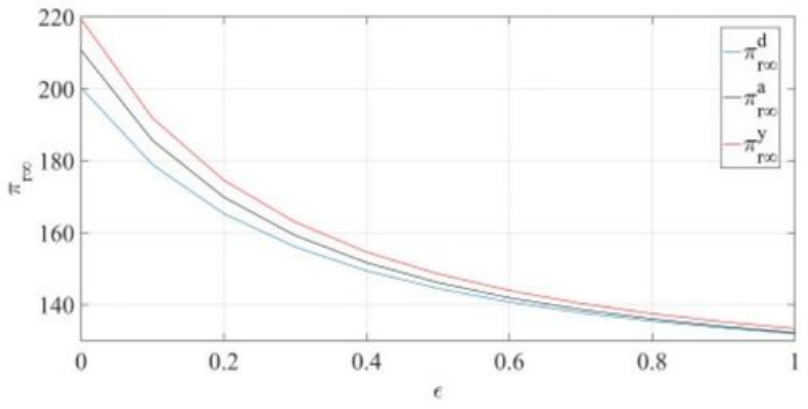

(b)

\section{Figure 6}

Please see the Manuscript PDF file for the complete figure caption 